Facing Humanness:

Facial Width-to-Height Ratio Predicts Ascriptions of Humanity

Jason C. Deska, E. Paige Lloyd, \& Kurt Hugenberg

Miami University

(C) 2017, American Psychological Association. This paper is not the copy of record and may not exactly replicate the final, authoritative version of the article. Please do not copy or cite without authors' permission. The final article will be available, upon publication, via its DOI:

$10.1037 /$ pspi0000110

Authors' note:

This research was supported by NSF grant BCS-1423765

Corresponding author: Jason C. Deska; deskajc@ miamioh.edu; Miami University, 90 North Patterson Avenue, Oxford, Ohio 45056

Author e-mail addresses: Jason C. Deska, deskajc@miamioh.edu; Kurt Hugenberg, hugenbk@miamioh.edu; E. Paige Lloyd, lloydep2@miamioh.edu 


\begin{abstract}
The ascription of mind to others is central to social cognition. Most research on the ascription of mind has focused on motivated, top-down processes. The current work provides novel evidence that facial width-to-height ratio (fWHR) serves as a bottom-up perceptual signal of humanness. Using a range of well-validated operational definitions of humanness, we provide evidence across five studies that target faces with relatively greater fWHR are seen as less than fully human compared to their relatively lower fWHR counterparts. We then present two ancillary studies exploring whether the fWHR-to-humanness link is mediated by previously established fWHR-trait links in the literature. Finally, three additional studies extend this fWHRhumanness link beyond measurements of humanness, demonstrating that the fWHR-humanness link has consequences for downstream social judgments including the sorts of crimes people are perceived to be guilty of and the social tasks for which they seem helpful. In short, we provide evidence for the hypothesis that individuals with relatively greater facial width-to-height ratio are routinely denied sophisticated, humanlike minds.

Keywords: facial width-to-height ratio; mind perception; face perception; dehumanization; impression formation
\end{abstract}




\section{Facing Humanness:}

\section{Facial Width-to-Height Ratio Predicts Ascriptions of Humanity}

Ascribing sophisticated minds to others is central to social cognition and behavior (Dennet, 1996). The so-called "other minds" problem - whether others have minds, and if so, of what faculties - is a long-standing question in philosophy (e.g., Wittgenstein \& Anscombe, 1958). Recently, psychologists too have begun investigating how and when perceivers ascribe minds to others. Despite differences in approach and focus, various psychological literatures investigating anthropomorphism (e.g., Epley, Akalis, Waytz, \& Cacioppo, 2008; Epley, Waytz, \& Cacioppo, 2007), dehumanization (e.g., Haslam, 2014; Haslam \& Loughnan, 2014), infrahumanization (e.g., Leyens, Paladino, Rodriguez-Torres, Vaes, Demoulin, Rodriguez-Perez, \& Gaunt, 2000; Leyens, Demoulin, Vaes, Gaunt, \& Paladino, 2007), objectification (Fredrickson \& Roberts, 1997; Gruenfeld, Inesi, Magee, \& Galinsky, 2008; Nussbaum, 1999), and mind perception (e.g., Gray, Gray, \& Wegner, 2007; Waytz, Gray, Epley, \& Wegner, 2010) all share a core interest in when sophisticated humanlike faculties are ascribed to, or withheld from, others.

The consequences of ascribing or withholding personhood can be profound. Ascribing humanity to others makes them part of the moral community, in which rights are protected and fair treatment is valued (Opotow, 1990). Indeed, seeing others as fully human facilitates empathy and the desire to help others in need (Cuddy, Rock, \& Norton, 2007). Conversely, withholding humanness can make others appear to have blunted emotional faculties (Leyens et al., 2007) and can trigger discrimination (Pereira, Vala, \& Leyens, 2009), aggression (Viki, Osgood, \& Phillips, 2013), and exacerbate intergroup conflict (see Haslam, 2014). 
Despite extensive developments in theory surrounding mind ascription (for reviews, see Epley, Schroeder, \& Waytz, 2013; Vaes, Bain, \& Leyens, 2014), most models of ascribing and withholding humanness focus extensively or even exclusively on top-down, motivated biases in the ascription of personhood, examining how beliefs about others (e.g., ingroup/outgroup distinctions) or current motives (e.g., desires to belong) influence whether others are seen as having fully human capacities. In the current work, we seek to extend the influence of this research tradition by focusing instead on a novel bottom-up bias in the ascription of humanness to others. Specifically, we test the hypothesis that a stable individual difference inherent in human faces - facial width-to-height ratio (fWHR) - predicts ascriptions of humanness to others.

To this end, we first provide a brief summary of the recent literature on ascribing and withholding humanity, before reviewing how facial width-to-height ratio influences person perception. We then summarize research pointing to the possible role of face perception in dehumanization. Finally, we present novel research testing whether faces high in fWHR, relative to faces low in fWHR, are seen as less than fully human. Across our first five studies, we use multiple well-established techniques to test whether faces high in fWHR are blatantly dehumanized, are ascribed less sophisticated minds, and are infrahumanized. We then present two ancillary studies exploring whether the fWHR-to-humanness link is mediated by previously established fWHR-trait links in the literature. In our final three studies, we test whether the lower levels of sophisticated human characteristics ascribed to high fWHR targets ramify throughout social judgments.

Considering mind ascription as the result of normal face processing mechanisms is, we believe, a distinctly novel theoretical contribution. The possibility that biases in ascriptions of 
humanness can result from targets' facial structural cues can point us toward surprising causes, consequences, and implications of dehumanization.

\section{On Ascribing Mind}

Whereas the consequences of dehumanization are troubling and have long been of interest to scholars (Kelman, 1976), the cognitive processes underlying ascribing and withholding personhood have only recently received greater scrutiny (see Bain, Vaes, \& Leyens, 2014). A lengthy review of the various theories of dehumanization and similar constructs is beyond the scope of the current work (for reviews, see Haslam \& Loughann, 2014; Waytz et al., 2010). However, there is some consistency in how scholars from multiple research traditions including the infrahumanization (Leyens, Demoulin, Vaes, Gaunt, \& Paladino, 2007), dehumanization (Haslam, 2006, 2014), and mind perception (Waytz et al., 2010) literatures explain ascriptions of humanity (see Bain et al., 2014 for a review). Indeed, all three prominent perspectives focus on how humans are seen as possessing sophisticated capacities that are distinct from other animals, while having an emotional responsiveness and experiential capacity that makes humans distinct from automata (see Haslam, 2014).

Non-human animals, such as dogs and frogs, are seen as able to experience the world and simple emotional states, but have limited cognitive and agentic abilities; non-human agents, such as robots, are seen as able to cogitate and act upon the world, but are believed to lack inner experience (Gray et al., 2007). Only (adult) humans are seen as having both sophisticated experiential and agentic capacities. Further, this distinction between "unthinking" animals and "unfeeling" machines is reflected in how people are dehumanized. Humans who are seen as being emotionally responsive and socially engaged, but lacking rationality and civility are seen as animal-like (i.e., animalistic dehumanization) whereas humans who are seen as rational and 
civil, but lacking in emotional responsiveness and interpersonal warmth are seen as machine-like (i.e., mechanistic dehumanization; see Haslam, 2006, 2014; Loughnan \& Haslam, 2007).

As noted above, perceivers tend to ascribe and withhold sophisticated minds when it suits them to do so. Multiple beliefs and motivations about the self and others, such as the desire for connectedness (Epley et al., 2008a, 2013; Epley, Waytz, Akalis, \& Cacioppo, 2008; Powers, Worsham, Freeman, Wheatley, \& Heatherton, 2014), the desire for control (e.g., Epley et al., 2008b; Waytz, Morewedge, Epley, Montelone, Gao, \& Cacioppo, 2010), or intergroup contexts and group-based stereotypes (Castano \& Giner-Sorolla, 2006; Goff, Eberhardt, Williams, \& Jackson, 2008; Leyens et al., 2000; Leyens et al., 2007; Vaes, Paladino, Castelli, Leyens, \& Giovanazzi, 2003), can influence the degree to which individuals ascribe personhood.

This robust relationship between intergroup motives and dehumanization, however appears to be more than mere intergroup prejudice. Although dehumanization and prejudice do often co-occur in vivo, dehumanization and prejudice are distinct concepts that are empirically separable and have been demonstrated to have unique consequences (e.g., Goff et al., 2008; Goff, Jackson, Di Leone, Culotta, \& DiTomasso, 2014; Haslam \& Loughnan, 2012; Kteily, Bruneau, Waytz, \& Cotterill, 2015). First, prejudice and dehumanization have distinct conceptual definitions. Whereas prejudice is a valenced global attitude toward a social group (Dovidio \& Gaertner, 1999), dehumanization is the denial of others' fully human faculties. Importantly, these are quite distinguishable in practice. Whereas one may dislike a group (e.g., professors, because of their diabolical midterms), they may still appear fully capable of thinking and feeling. Similarly, one may love a group, but still see them as incapable of acting rationally (e.g., toddlers). Even individuals who are highly motivated toward prosocial behaviors - such as 
surgeons operating on patients - dehumanize those they are helping in service of the helping itself (Haque \& Waytz, 2012).

Further, prejudice and dehumanization can elicit separable empirical predictions and result in distinct consequences. Whereas prejudice yields, by definition, a valenced prediction (i.e., negative groups are ascribed negative traits or outcomes), dehumanizing a group can lead to the reduction in perceptions of both positive and negative aspects of mind. For example, in research on infrahumanization, outgroups are often seen as being less capable of feeling both negative and positive sophisticated emotions - both nostalgia and ennui are reserved for fully humanized ingroups, despite the fact that one emotion is evaluatively positive and one is evaluatively negative. Similarly, dehumanized groups or individuals are commonly ascribed both less positive humanlike faculties (e.g., broadminded) and less negative humanlike faculties (e.g., stingy; Haslam \& Bain, 2007). More recently, work by Kteily and colleagues (2015) shows that blatant dehumanization effects predict important social judgments and occur even when statistically controlling for prejudice. Taken together, although real world cases of dehumanization are often most troubling when the outgroup is disliked, dehumanization is both conceptually and empirically distinct from prejudice.

\section{Humanness from Faces: The Case for Facial Width-to-Height Ratio}

As noted above, most previous dehumanization research has focused on top-down individual and group-related motives. Yet, of growing interest is how and when perceivers infer mind in a bottom-up manner, triggered by cues in others' faces and bodies. There is ample evidence that the presence of humanlike facial features (e.g., more human faces in a doll-tohuman morph continuum; Looser \& Wheatley, 2010) and humanlike configurations (e.g., upright but not inverted faces; Deska, Almaraz, \& Hugenberg, 2017; Hugenberg et al., 2016) are 
spontaneously experienced as human and are ascribed humanlike minds, traits, and characteristics.

However, this small literature has been, as yet, mute on which facial dimensions signal humanness, or the lack thereof. Although we know that the mere presence of humanlike features (e.g., eyes) or humanlike configurations (i.e., eyes-over-nose-over-mouth) is sufficient to trigger the experience that an agent has humanlike faculties, it remains unclear which naturally occurring dimensions of faces signal humanness (or its absence). Thus, the current work builds on these past demonstrations of bottom-up signals of humanness. However, rather than providing a non-human agent with cues of humanness (e.g., adding human eyes to a doll; Balas \& Tonsager, 2014), or disrupting the cues of humanness in faces (e.g., by morphing with dolls, or by inverting faces), we investigate how features that naturalistically vary among human faces may be differentially associated with humanness. Specifically, we focus on a stable characteristic of faces, facial width-to-height ratio.

Facial width-to-height ratio is a structural feature of faces that exists independent of facial musculature and, as shown in Figure 1, is commonly measured as the ratio between bizygomatic width (i.e., distance between left to right zygion) and upper face height (i.e., distance between mid-brow and upper lip; Hehman, Flake, \& Freeman, 2015). Initial work indicated that fWHR might be sexually dimorphic (Weston, Friday, \& Liò, 2007) and linked to pubertal testosterone in men (e.g., Lefevre, Lewis, Perrett, \& Penke, 2013); however, more recent work has found little evidence for this putative relationship between fWHR and sex or testosterone (Bird et al., 2016; Hodges-Simeon, Sobraske, Samore, Gurven, \& Gaulin, 2016; Kramer, Jones, \& Ward, 2012; Lefevre, Etchells, Howell, Clark, \& Penton-Voak, 2014; Özener, 2012), suggesting that high versus low fWHR is likely not a sex typical feature. 
Regardless of the origin, natural variation in fWHR predicts both physical and social aggression. For example, people higher in fWHR (but not higher in facial masculinity) selfreport as being more aggressive and behaviorally dominant than do lower fWHR individuals (Lefevre et al., 2014). Further, this self-reported aggression appears to be mirrored in actual behavior. For example, high fWHR hockey players compete more aggressively than their lower fWHR counterparts (operationalized as time spent in the penalty box; Carré \& McCormick, 2008; cf. Deaner, Goetz, Shattuck, \& Schnotala, 2012). Similarly, fWHR is positively associated with both estimated and actual aggression of men engaged in competitive tasks (Carré, McCormick, \& Mondloch, 2009). Facial width-to-height ratio also predicts neural responses to stressors. Carré, Murphy, and Hariri (2013) found that amygdala reactivity to angry faces predicted aggression among high-fWHR men but not among low-fWHR men. Social aggression is also associated with higher fWHR. Work by Hehman, Leitner, Deegan, and Gaertner (2013) demonstrated that White men with relatively higher fWHR demonstrated more overt racial animus, an important component of intergroup conflict. Further, higher fWHR men are more likely to exploit the trust of others or cheat for selfish gain (e.g., Geniole et al., 2014; Stirrat \& Perrett, 2010). Finally, fWHR behavioral effects are robust across studies; a meta-analytic review of 18 studies shows a reliable effect relating fWHR to behavioral aggression (Haselhuhn, Ormiston, \& Wong, 2015).

More germane to the current work, fWHR does not just predict behavior, but also influences judgments of others. Mirroring the behavioral data, targets higher in fWHR are judged to be more aggressive than their lower fWHR counterparts. Carré and colleagues (2009) showed people images of men displaying neutral expressions and asked them to estimate how aggressive the target would be if provoked. Across two studies, targets' fWHR was positively associated 
with perceptions of aggression, even at extremely quick presentation times (i.e., $39 \mathrm{~ms}$ ). Short and colleagues (2012) demonstrated that these judgmental effects occur for both adult and child perceivers, and for own-race and other-race targets, suggesting that fWHR biases judgments independently of age and race. Recent studies of CEOs have found that perceivers associate higher fWHR executives with greater trait dominance (Alrajih \& Ward, 2014; see also Mileva et al., 2014). Moreover, relatively high fWHR faces facilitate the recognition of, and bear structural similarity to, anger expressions (e.g., Deska, Lloyd, \& Hugenberg, in press; Neth \& Martinez, 2010). Reifying the robustness of these fWHR effects on judgment, Geniole and colleagues' (2015) meta-analytic review reveals that individuals with greater facial width-to-height ratios are reliably perceived as more threatening and dominant.

The effects of fWHR on person perception have important consequences for social judgments, but not always to the detriment of high fWHR targets. In some contexts, high fWHR targets are preferred. For example, perceivers prefer to select high fWHR targets as group members in competitive intergroup contexts (Hehman, Leitner, Deegan, \& Gaertner, 2015). However in other contexts, such as criminal sentencing, high fWHR targets are punished. Work by Wilson and Rule (2015) analyzed the faces and sentencing decisions of 742 men convicted by the State of Florida for murder, and found that higher fWHR convicts were more likely to be sentenced to die than to spend life in prison, perhaps a surprising implication for a facial structural cue that perceivers may be unaware is biasing judgments at all.

\section{Current Research: Facial Width-to-Height Ratio and Dehumanization}

Taken together, the extant literature paints a picture of the high fWHR individual as someone who is impulsively aggressive and generally unsociable (Anderl et al., 2016), a combination of characteristics that appears to overlap with perceptions of individuals who lack 
human sophistication - mapping closely onto multiple models of biases in mind ascription (i.e., mind perception, Gray et al., 2007; senses of humanness, Haslam, 2006; blatant dehumanization, Kteily et al., 2015; infrahumanization, Leyens et al., 2000). From this, it is possible that these established fWHR-to-trait links may actually generate the perception that higher fWHR targets are less than fully human (relative to their lower fWHR counterparts).

We see the possibility of a reliable, yet unarticulated link between facial width-to-height ratio and humanness existing under our noses (or perhaps slightly above our noses) as a particularly provocative hypothesis for a number of reasons. First, the current work distinguishes itself from most research on dehumanization because of its focus on bottom-up signals that exist in faces, rather than on top-down motives that exist in perceivers. Second, the current work focuses on a naturally occurring structural component of faces rather than manipulating the presence or absence of human features in stimuli (e.g., Looser \& Wheatley, 2010) or inserting cues of humanness into otherwise non-human faces (e.g., Balas \& Horski, 2012). Thus, we propose that in spite of the fact that all of the targets in our work actually are human, a naturally varying face structure differentially signals humanness.

In the current work, we test whether high fWHR individuals are denied full humanness relative to low fWHR individuals. We had three primary goals. The first was to test whether the fWHR-to-humanness link occurs across a variety of measures of humanness. In Studies 1-5, we sought to test this hypothesis by using four of the most widely used and accepted measures of humanness including measures of blatant dehumanization (Kteily et al., 2015), mind perception (Gray et al., 2007), infrahumanization (Cortes, Demoulin, Rodriguez, Rodriguez, \& Leyens, 2005), and dehumanization (Haslam \& Bain, 2007). Our second goal was to investigate the process by which fWHR may influence inferences of humanness. Two Ancillary Studies, 
presented between Study 5 and Study 6, test whether the fWHR-to-humanness link is mediated by existing fWHR-trait relationships observed in the existing literature (e.g., dominance).

Finally, our third goal was to explore the downstream consequences of the fWHR-to-humanness link. In Studies 6-8, we sought to examine its consequences for beliefs about criminality and the suitability of individuals for social roles, as well as expectations about success across multiple life domains. Across these studies, we find evidence supporting the hypothesis that high fWHR individuals are denied full humanness, that it may be mediated through a constellation of theoretically consistent person perception variables, and that it has robust downstream consequences.

\section{Studies 1-5: Investigating the existence of a fWHR-humanness link}

\section{Study 1a}

Recently, Kteily and Bruneau (in press) argued that overt dehumanization is surprisingly commonplace and can uniquely predict deleterious attitudes and behaviors over and above subtle measures of dehumanization. To that end, we adapted Kteily and colleagues' (2015) measure of blatant dehumanization for use in these opening experiments. In this procedure, participants are shown the classic "Ascent of Man" graphic with five silhouettes of a hominid varying in evolutionary stage, ranging from dryopithecus, an early human ancestor that bears striking similarities with apes, to modern day Homo sapiens. Participants are asked to indicate, using a slider scale beneath the Ascent of Man graphic, how evolved different targets are. Blatant dehumanization occurs for a variety of target groups, is separable from prejudice, and is more strongly associated with support for hierarchy, torture, and retributive violence against outgroups than more subtle measures of dehumanization (Kteily et al., 2015; Kteily, Hodson, \& Bruneau, 
2016). Thus, exploring the extent to which fWHR influences blatant dehumanization serves as an ideal preliminary test of the hypothesis.

In Study 1a, we sought to test whether high fWHR targets, relative to their low fWHR counterparts, are blatantly dehumanized. To test this, participants were shown a series of faces that varied in their fWHR, and were asked to complete the blatant dehumanization measure for each face. We hypothesized that high fWHR targets would be seen as less evolved than low fWHR targets, indicative of blatant dehumanization.

\section{Method}

Statistical Power and Participants. Because we were uncertain as to the effect sizes we may observe in the current work, we relied on the sample size of the most analogous study in the literature, which was Hugenberg and colleagues' (2016; Study 1) demonstration that configural processing can serve as a bottom-up signal of humanness. That study employed 51 participants. Therefore, we targeted approximately 50 participants in each of our studies, except as noted. Nevertheless, there was some variation in availability of participants across studies, leading to per-study samples slightly lower and higher than 50 across some of the studies. No analyses were conducted before data collection was complete in each study. No participants were excluded from analyses in any study. Forty-nine mTurk workers $\left(M_{\text {age }}=35.88, S D=10.35\right)$ completed Study 1a and were paid $\$ 0.40$. The majority of participants self-identified as White $(77.6 \%)$ and as male $(59.2 \%)$.

Materials. Twenty images of White men from the Chicago Face Database (CFD; Ma, Correll, \& Wittenbrink, 2015) were used as stimuli in this study. We employed faces from the CFD for several reasons. First, the CFD is a thoroughly normed and highly controlled set of face images. Second, and equally important, across the entire population of White male faces in the 
CFD, attractiveness does not correlate with fWHR, $r(91)=-.04, p=.703$, making it difficult to attribute any fWHR-based effects to simple valence halo effects.

We used the 10 faces of White males who had the highest facial width-to-height ratio ( $M$ $=2.04, S D=0.06)$ and 10 faces of White men who had the lowest fWHR $(M=1.74, S D=$ 0.03). ${ }^{1}$ Pretesting revealed that the high and low fWHR groups differed significantly on fWHR, $t(12.84)=14.46, p<.001, d=8.07 .^{2}$ Mirroring the overall lack of relationship between fWHR and attractiveness in the CFD overall, the high and low fWHR groups of selected faces did not differ on attractiveness $(M s=2.70$ vs. $2.89, S D s=0.49$ vs. 0.62$), t(18)=-0.79, p=.440, d=-$ 0.37. Images were resized to $682 \times 480$ pixels and were presented in color. All stimuli were displayed onscreen for 500ms. Presentation order was randomized separately for each participant.

Procedure. Participants completed all tasks online. After providing informed consent, participants were instructed that "people can vary in how human-like they seem. Some people seem highly evolved whereas others seem no different from lower animals." They were then informed that they would see a series of briefly displayed faces, and that they would answer questions about each face. Participants were then shown the 20 target faces (10 high fWHR; 10 low fWHR) in a random sequence. Participants were asked to indicate how evolved each target seemed using a sliding scale paired with the Ascent of Man image (Kteily et al., 2015). Finally,

\footnotetext{
${ }^{1}$ We used the following stimuli from the Chicago Face Database v1.0: WM-039; WM-011; WM-001; WM-009; WM-036; WM-003; WM-018; WM-023; WM-025; WM-010; WM-022; WM-032; WM-035; WM-038; WM-006; WM-021; WM-016; WM-040; WM-041; \& WM-019. ${ }^{2} \mathrm{We}$ acknowledge that there is some disagreement on how effect size should be calculated for repeated-measures samples. For this and all future effects, we calculated Cohen's $d$ using pchecker app created by Felix Schönbrodt, http://shinyapps.org/apps/p-checker/.
} 
participants provided basic demographic information (e.g., sex, age, race), were debriefed, and were provided their compensation code.

\section{Results}

Repeated-measures designs in which participants evaluate samples of stimuli can inflate Type 1 error rates when analyzed with ANOVAs (Judd, Westfall, \& Kenny, 2012; Judd, Westfall \& Kenny, 2017). Consequently, in this and all studies, we use mixed-model analyses that fully crossed participants and stimuli and treated both as random factors. Facial width-to-height ratio was effect coded in all studies (low fWHR $=-0.5$, high fWHR $=0.5$ ).

Consistent with our hypothesis that high fWHR targets are dehumanized compared to low fWHR targets, we observed a significant effect of fWHR, $B=-4.23, S E=1.74,95 \%$ CI [-7.86, 2.43], $t(20.05)=-2.45, p=.025, d=-1.08$, such that high fWHR targets $(M=80.64, S D=18.05)$ were rated as significantly less evolved than low fWHR targets $(M=84.89, S D=15.17)$.

\section{Discussion}

Study 1a provides initial evidence that high fWHR targets are perceived as less evolved than low fWHR targets, an effect consistent with blatant dehumanization (Kteily et al., 2015; Kteily et al., 2016). High fWHR individuals are seen as more akin to our evolutionary ancestors than low fWHR individuals.

\section{Study 1b}

Study 1a employed only male faces as stimuli, as is common in the majority of research studies on fWHR (Geniole et al., 2015; Haselhuhn et al., 2015). Nonetheless, female faces also naturalistically vary in fWHR and recent research has found that the effects of fWHR on judgments of female faces have effects quite similar to judgment of male faces (Deska et al., in press). Thus, it is possible that fWHR might bias judgments of humanity similarly for both male 
and female targets. Study $1 \mathrm{~b}$ was designed to test this possibility. Participants completed the same task as participants in Study 1a, with the addition of White female faces. If fWHR functions similarly in female faces as it does in male faces we would expect a main effect of fWHR, replicating Study 1a. If fWHR does not signal humanness in female faces as it does in male faces, then an interaction between fWHR and sex should occur. Further, using the same male faces in Study $1 \mathrm{~b}$ as in Study 1a allows us to provide a replication of the fWHR-based blatant dehumanization effect for male faces.

\section{Method}

Participants. Fifty mTurk workers $\left(M_{\text {age }}=36.50, S D=12.72\right)$ completed this study and were paid $\$ 0.50$ for their participation. The majority of participants self-identified as White $(82.0 \%)$ and as female $(52 \%)$.

Materials and Procedure. The materials and procedure for Study $1 \mathrm{~b}$ were identical to Study 1a except as noted. In addition to responding to the blatant dehumanization measure for the 20 male faces, participants also completed this measure for 20 female faces. ${ }^{3}$ We used the same selection criteria for the 20 female faces as we did for the male faces: we chose the 10 faces of White females who had the highest facial width-to-height ratio $(M=2.08, S D=0.10)$ and 10 faces of White females who had the lowest fWHR $(M=1.77, S D=0.90)$. Pretesting revealed that these high and low fWHR female face groups differed significantly on fWHR, $t(18)$ $=7.51, p<.001, d=3.54$. In the full CFD, unlike White male faces, fWHR and attractiveness are marginally related for White female faces, $r(88)=-.20, p=.059$. However, in our selected

\footnotetext{
${ }^{3}$ We used the following stimuli from the Chicago Face Database: WF-024; WF-025; WF-028; WF-034; WF-012; WF-014; WF-013; WF-018; WF-006; WF-021; WF-031; WF-035; WF-036; WF-017; WF-029; WF-010; WF-033; WF-037; WF-002; \& WF-007.
} 
subset, face groups did not significantly differ on attractiveness $(M s=2.99$ vs. $3.58, S D s=0.78$ vs. 0.81$), t(18)=1.63, p=.121, d=0.77$ (although the effect size is large for a non-significant comparison, suggesting a lack of power). There was not a significant fWHR by target sex interaction on attractiveness, $F(1,36)=0.78, p=.383, \eta_{p}^{2}=.02$. Images were resized to $682 \times 480$ pixels and were presented in color.

\section{Results}

Of primary interest in the current study was the extent to which male and female high fWHR targets were blatantly dehumanized, relative to their low fWHR counterparts. Target sex was effect coded $($ male $=-0.5$, female $=0.5)$. Consistent with our predictions, we observed a main effect of fWHR, $B=-4.51, S E=1.76,95 \%$ CI $[-8.05,-0.96], t(43.37)=-2.56, p=.014, d=$ -0.78. High fWHR targets $(M=74.89, S D=18.25)$ were rated as significantly less evolved than their low fWHR counterparts $(M=79.39, S D=15.91)$. There was not a significant effect of target sex, $B=3.09, S E=1.85,95 \%$ CI $[-0.62,6.80], t(50.37)=1.67, p=.100, d=0.47$. Male targets $(M=75.59, S D=18.09)$ were seen as equivalently evolved as female targets $(M=78.69$, $S D=16.34)$. We did not observe a fWHR by target sex interaction, $B=-0.31, S E=3.39,95 \% \mathrm{CI}$ $[-7.18,6.56], t(38.37)=-0.09, p=.929, d=-0.03$.

\section{Discussion}

The purpose of Study 1b was twofold. First, we sought to investigate whether the effects of fWHR on blatant dehumanization are specific to male faces or if fWHR functions similarly for both male and female faces. Second, we sought to replicate the findings of Study 1a. We observed only an unqualified main effect of fWHR, replicating Study 1a and suggesting that fWHR may signal humanness similarly in male and female faces.

\section{Study 1c}


Extensive past work has demonstrated that race has robust effects on face and person perception (Blair, Judd, \& Fallman, 2004; Freeman \& Ambady, 2011; Golby, Gabrieli, Chiao, \& Eberhardt, 2001; Goff et al., 2008; see Hugenberg \& Wilson, 2013 for a review). Because of this, we intentionally held race constant in Studies 1a and 1b. Nevertheless, because past work has shown that the effects of fWHR on perceptions of aggression generalize across race (Short et al., 2012), we hypothesized that fWHR might signal humanness similarly across racial boundaries. To investigate this directly, in Study 1c, participants completed the same task as Study 1a with the addition of Black male targets. Of primary interest was whether the effects of fWHR were observed equivalently across target race. By using the same White male faces in Study 1c as in Study 1a, the current Study provided another opportunity for replication of the fWHR-based blatant dehumanization effect among the White male faces.

\section{Method}

Participants. Forty-nine mTurk workers $\left(M_{\text {age }}=43.78, S D=13.32\right)$ completed this study and were provided $\$ 0.50$ for their participation. The majority of participants self-identified as White $(85.7 \%)$ and as female $(53.1 \%)$.

Materials and Procedure. The materials and procedure for the current study were identical to Study 1a except as noted. In addition to responding to the blatant dehumanization measure for the 20 White faces, participants also completed this measure for 20 Black faces. ${ }^{4} \mathrm{We}$ used the same selection criteria for Black male faces as we did for the White male faces. Specifically, we chose the 10 faces of Black males who had the highest fWHR $(M=2.02, S D=$

\footnotetext{
${ }^{4}$ We used the following stimuli from the Chicago Face Database: BM-018; BM-024; BM-022; BM-037; BM-041; BM-026; BM-043; BM-013; BM-017; BM-019; BM-003; BM-010; BM-046; BM-044; BM-038; BM-016; BM-045; BM-011; BM-028; \& BM-032.
} 
$0.04)$ and 10 faces of Black males who had the lowest fWHR $(M=1.75, S D=0.05)$. These high and low fWHR groups differed significantly on fWHR, $t(18)=12.89, p<.001, d=6.08$. The high $(M=2.99, S D=0.47)$ and low $(M=3.70, S D=0.69)$ fWHR Black male faces did differ in attractiveness, $t(18)=-2.67, p=.016, d=-1.26$, but there was not a fWHR by target race interaction, $F(1,36)=1.96, p=.170, \eta_{p}^{2}=.05$.

\section{Results}

Of primary interest was the extent to which Black and White high fWHR targets were seen as less evolved than their low fWHR counterparts. Target race was effect coded (White $=-$ 0.5 , Black $=0.5)$. We did not observe a main effect of fWHR, $B=-2.85, S E=1.76,95 \%$ CI [$6.86,1.17], t(8.50)=-1.62, p=.142, d=-1.11$. High fWHR targets $(M=79.99, S D=16.93)$ were rated as equivalently evolved as their low fWHR counterparts $(M=82.84, S D=15.36)$. We did observe a significant main effect of target race, $B=-6.34, S E=2.71,95 \%$ CI [-11.85, -0.82], $t(32.97)=-2.34, p=.026, d=-0.81$. Black targets $(M=78.25, S D=21.38)$ were rated as significantly less evolved than White targets $(M=84.59, S D=13.18)$. We did not observe a fWHR by target race interaction, $B=0.06, S E=3.32,95 \% \mathrm{CI}[-7.87,7.98], t(6.69)=0.02, p=$ $.987, d=0.01$.

\section{Discussion}

Contrary to predictions, we did not observe an effect of facial width-to-height ratio on ascription of humanity in Study 1c, nor did we observe an interaction between target race and fWHR. We did, however, observe that our primarily White participants rated Black targets as less evolved than White targets. This effect could be due to culturally bound stereotypes associating Blacks with animals (e.g., Goff et al., 2008). Alternatively, it could be a result of group processes, as participants were primarily White. Indeed, infrahumanization theory (Leyens 
et al., 2000) suggests that outgroups are denied the human essence relative to ingroup members. Nevertheless, despite not reaching statistical significance, the effects were in the predicted direction.

\section{Study 1d}

Given the results of Studies 1a and b, we were surprised not to observe an effect of fWHR on humanness in Study 1c, in spite of the results being descriptively consistent with the previous findings. To provide a more robust understanding of the phenomenon, Study $1 \mathrm{~d}$ served as a direct replication of Study 1c.

\section{Method}

Participants. Fifty-three mTurk workers $\left(M_{\text {age }}=35.30, S D=11.54\right)$ completed this study and were provided $\$ 0.50$ for their participation. The majority of participants self-identified as White $(75.0 \%)$ and $45.3 \%$ self-identified as female.

Materials and Procedure. The materials and procedure for the current study were identical to Study 1c.

\section{Results}

Of primary interest was the extent to which Black and White high fWHR targets were seen as less evolved than their low fWHR counterparts. We observed a main effect of fWHR, $B$ $=-3.00, S E=0.92,95 \%$ CI $[-4.84,-1.16], t(64.53)=-3.26, p=.002, d=-0.81$. High fWHR targets $(M=79.99, S D=16.93)$ were rated as less evolved than their low fWHR counterparts $(M$ $=82.84, S D=15.36)$. We also observed a significant main effect of target race, $B=-8.44, S E=$ $3.01,95 \%$ CI $[-14.48,-2.41], t(55.84)=-2.80, p=.007, d=-0.75$. Black targets $(M=78.25, S D$ $=21.38)$ were rated as significantly less evolved than White targets $(M=84.59, S D=13.18)$. We 
did not observe a fWHR by target race interaction, $B=1.56, S E=1.57,95 \%$ CI [-1.59, 4.71], $t(50.34)=1.00, p=.324, d=0.28$.

\section{Discussion}

Consistent with Studies 1a and 1b, in Study 1d, participants ascribed less humanity to high fWHR targets compared to low fWHR targets. Replicating Study 1c, we again observed a main effect of target race such that Black targets were seen as less human than White targets. However, and consistent with Study 1c, we did not observe a fWHR by target race interaction, suggesting that fWHR may operate similarly on White and Black targets.

Together, Studies 1a-1d provide evidence consistent with the hypothesis that, compared to their low fWHR counterparts, high fWHR faces are blatantly dehumanized. However, given the inconsistencies across Studies 1c and 1d, we conducted a small meta-analysis (Goh, Hall, \& Rosenthal, 2016) of Studies 1a-1d. This analysis showed that the effect of fWHR on blatant dehumanization is reliable, $r=0.43,95 \% \mathrm{CI}[0.31,0.54], z=6.03, p<.001$. As noted above, the blatant dehumanization scale is face-valid and tends to correlate more strongly with support for hierarchy, torture, and retributive violence than subtler measures of dehumanization (Kteily et al., 2015; Kteily et al., 2016). These studies also provide evidence suggesting that the effects of fWHR on blatant dehumanization may generalize to White female and Black male targets.

\section{Study 2}

Although studying blatant dehumanization may be particularly important given the relationship to real-world outcomes (e.g., Kteily et al., 2016), it remains an open question as to whether these effects generalize to subtler measures of humanity. The primary goal of Study 2 was to test whether fWHR influences ascriptions of agency and experience. In their influential model, Gray and colleagues (2007) demonstrated that people perceive others' minds as being 
comprised of two distinct and conceptually orthogonal dimensions: agency (i.e., the capacity for higher order cognitions, such as planning, communicating, and remembering) and experience (i.e., the capacity for experiencing sensations and drive-states such as pleasure, pain, and hunger). Individuals high in agency can act with intention and thus are seen as moral agents, capable of enacting right and wrong, whereas individuals high in experience are seen as moral patients, capable of being acted upon (Gray, Knobe, Sheskin, Bloom, \& Barrett, 2011; Gray \& Wegner, 2010; Gray, Young, \& Waytz, 2012). Because high fWHR targets are often seen as impulsively aggressive (Geniole et al., 2015), we hypothesized that they would be ascribed minds that are low in agency. Our predictions for the experience dimension were less clear. It seemed unlikely that adult human targets would be fully denied basic experiential drive states hunger, fear, and the capacity to feel pain and pleasure - leading to the possibility that high fWHR faces would be denied agency but not experience (much like an animal). However, given that recent research has demonstrated that not all adult humans are seen equally capable of basic experiences such as pain (e.g., Trawalter, Hoffman, \& Waytz, 2012), it was also plausible that high fWHR faces would be denied both agency and experience.

To investigate the hypothesized link between targets' fWHR and mind ascription, participants completed a procedure very similar to Study 1a, except that here participants rated high and low fWHR target faces on dimensions of agency and experience (Gray et al., 2007).

\section{Method}

Participants. Forty-seven mTurk workers $\left(M_{a g e}=34.36, S D=11.87\right)$ were paid $\$ 0.40$ to participate in this study. Most participants identified as White (78.7\%) and male $(55.3 \%)$.

Materials and Procedure. The materials and procedure for the current study were identical to Study 1a except as noted. After seeing each face, participants responded to 8 items 
adapted from Gray and colleagues' (2007) measures assessing perceived agency (i.e., This person is capable of exercising self-control, This person has a good memory, This person is capable of acting morally, This person is capable of recognizing emotions; $\alpha=.95)$ and experience (i.e., This person is capable of feeling hungry, This person is capable offeeling fear, This person is capable of feeling pain, This person is capable of feeling pleasure; $\alpha=.97)$. These items were selected because they were the four that loaded most strongly onto the agency and experience factors in the original work (Gray et al., 2007; see also Cooley, Payne, Cipolli, \& Gray, 2017). In our data, agency and experience were positively correlated, $r(45)=.41, p=.005$.

\section{Results}

Of primary interest in the current study was the extent to which target fWHR biased ascriptions of agency and experience. Mind dimension was effect coded (agency $=-0.5$, experience $=0.5)$ We observed a significant effect of mind dimension such that all faces were ascribed more experience $(M=5.62, S D=0.83)$ than agency $(M=4.94, S D=0.75), B=0.63$, $S E=0.14,95 \%$ CI $[0.38,0.88], t(44.19)=5.07, p<.001, d=1.53$. We did not observe a significant main effect of fWHR. Faces high in fWHR were not ascribed less mind $(M=5.21$, $S D=0.72)$ than faces low in fWHR overall $(M=5.35, S D=0.64), B=-0.15, S E=0.14,95 \% \mathrm{CI}$ $[-0.42,0.13], t(466.56)=-1.06, p=.289, d=-0.10$. However, we observed a significant interaction between fWHR and mind dimension, $B=0.18, S E=0.07,95 \%$ CI $[0.04,0.32]$, $t(1918.03)=2.60, p=.009, d=0.12$ (see Figure 2$)$. We decomposed this interaction by comparing the effect of fWHR separately for agency and experience. High fWHR targets $(M=$ 4.80, $S D=0.82)$ were ascribed marginally less agency than were their low fWHR counterparts $(M=5.04, S D=0.68), B=-0.24, S E=0.13,95 \%$ CI $[-0.51,0.03], t(23.06)=-1.81, p=.083, d=$ -0.75; however, low and high fWHR targets did not differ in their ascribed experience $(M \mathrm{~s}=$ 
3.80 vs. $3.90 ; S D$ s $=0.87$ vs. 0.83$), B=-0.06, S E=0.06,95 \%$ CI $[-0.18,0.07], t(27.38)=-0.96$, $p=.344, d=-0.37$.

\section{Discussion}

In Study 2, we found that high fWHR targets were denied sophisticated, agentic minds compared to their low fWHR counterparts. Notably, however, high and low fWHR targets were not ascribed a differential capacity to experience. Consistent with perceptions of high fWHR targets as aggressive, threatening, and dominant (e.g., Geniole et al., 2015), high fWHR targets were denied only traits indicative of mental sophistication. Yet, high fWHR targets were still perceived as capable of feeling. Indeed, even entities such as sea slugs appear to experience drive states such as hunger and pain - it perhaps takes too great an act of mental gymnastics to deny these experiential states to targets who, despite varying in facial structure, are obviously all adult humans (cf. Trawalter et al., 2012). Nevertheless, the current study provides evidence that, using a well-established measure of mind ascription, high fWHR targets are ascribed less sophisticated minds than are low fWHR targets.

\section{Study 3a}

Studies $3 \mathrm{a}$ and $3 \mathrm{~b}$ were designed to extend the link between fWHR and humanness to another well-established set of human faculties: emotional experiences seen as unique to humans. At the heart of infrahumanization theory is the premise that sophisticated secondary emotions are reserved for the ingroup (Leyens et al., 2000). Although many creatures may feel primary emotions such as happiness or sadness, people often reserve secondary emotions, such as pride or remorse, for the ingroup. From the perspective of infrahumanization theory, it is this distinction between simple basic emotions and sophisticated secondary emotions that signals targets are not being perceived as fully human. Here, we sought to extend the findings of the 
previous studies to infrahumanizing judgments of individuals across fWHR. We hypothesized that high fWHR targets may be infrahumanized - denied complex emotions relative to basic emotions - compared to low fWHR targets who should be perceived as capable of experiencing the full range of emotions.

\section{Method}

Participants. Fifty-one mTurk workers $\left(M_{\text {age }}=37.06, S D=14.39\right)$ completed this study and were paid $\$ 0.50$. The majority of participants self-identified as White (84.3\%) and female $(51.0 \%)$

Materials and Procedure. The materials and procedure for Study 3 a were identical to Study 1a except as noted. After seeing each face, participants responded to 12 infrahumanization items (Cortes et al., 2005). Participants were asked to report the extent to which targets were capable of feeling six primary emotions (i.e., happiness, pleasure, passion, aversion, anger, irritation; $\alpha=.96$ ), and six secondary emotions (i.e., contentment, delight, enjoyment, melancholy, resignation, disarray; $\alpha=.97)$. Participants reported agreement that each target was capable of feeling each emotion on a 7-point scale, ranging from 1 (strongly disagree) to 7 (strongly agree).

\section{Results}

Of primary interest in the current study was whether high fWHR faces were infrahumanized relative to low fWHR faces. Emotion type was effect coded (primary $=-0.5$, secondary $=0.5)$. There was not a significant effect of emotion type, $B=-0.04, S E=0.04,95 \%$ CI $[-0.17,0.10], t(2.63)=-0.94, p=.426, d=-1.16$. Faces were ascribed equivalent levels of primary $(M=5.62, S D=0.83)$ and secondary $(M=4.94, S D=0.75)$ emotions. There was also not a significant main effect of fWHR. Faces high in fWHR were not ascribed less emotions $(M$ 
$=5.21, S D=0.72)$ than faces low in fWHR $(M=5.35, S D=0.64), B=0.00 S E=0.10,95 \% \mathrm{CI}$ $[-0.20,0.20], t(102.74)=0.011, p=.991, d=0.00$. However, we did observe the predicted interaction between fWHR and emotion type, $B=-0.10, S E=0.05,95 \%$ CI [-0.20, -0.01$]$, $t(1627.74)=-2.24, p=.025, d=-0.11$ (see Figure 3$)$. To decompose this interaction, we looked at the effect of emotion type separately for high and low fWHR targets. Participants believed that low fWHR targets could experience secondary emotions $(M=5.38, S D=0.91)$ and primary emotions $(M=5.37, S D=0.96)$ equivalently, $B=0.01, S E=0.03,95 \%$ CI $[-0.05,-0.08], t(50)=$ $0.45, p=.657, d=0.13$. However, participants believed that high fWHR targets had less capacity to experience secondary emotions $(M=5.33, S D=0.92)$ than primary emotions $(M=5.42, S D=$ 0.92), $B=-0.09, S E=0.04,95 \%$ CI $[-0.16,-0.01], t(50)=-2.46, p=.017, d=-0.70$.

\section{Discussion}

Study 3a provided evidence suggesting that high fWHR targets are infrahumanized relative to low fWHR targets (Leyens et al., 2000). Specifically, high fWHR targets were seen as less capable of experiencing complex secondary emotions than simple primary emotions.

However, low fWHR targets were seen as having equivalent capacity to experience both secondary and primary emotions. In other words, low fWHR faces simply appear to have more sophisticated emotional capacity than do high fWHR faces. Thus, this study provides further evidence for the hypothesis that high fWHR targets are systematically denied their full humanity. Past research on infrahumanization has focused extensively on how intergroup processes elicit group-based biases (Leyens et al., 2000; Leyens et al., 2007). The current research builds on this past work by demonstrating that infrahumanization can also be the result of bottom-up signals in the faces of others.

\section{Study 3b}


To this point, each study has used the same stimuli from the Chicago Face Database (Ma et al., 2015). Although employing such a highly standardized stimulus set has clear advantages, of interest in Study 3b was whether the infrahumanization effects from Study 3a would replicate using a very different set of stimuli: faces of convicted murderers. In Study 3b, we sought to replicate the effects of fWHR on infrahumanization using mugshots of men who had been incarcerated by the State of Florida for murder (Wilson \& Rule, 2015). Although these stimuli are also controlled in some ways (e.g., similar lighting; similar prison jumpsuits), these stimuli represent a distinct population from the volunteers in the CFD. Further, these targets allow us to investigate whether the effects of fWHR on humanness remain even amongst targets who occupy an unequivocally dehumanized role: prisoner. Given that the targets in Study 3b were wearing prison jumpsuits, we predicted that they would be generally infrahumanized (i.e., seen as more capable of primary than secondary emotions). The core question was whether high fWHR faces would be seen as less capable of secondary emotions than primary emotions compared to low fWHR faces, an effect consistent both with infrahumanization theory and with Study 3a.

\section{Method}

Participants. In Study 3b, any given participant viewed 20 of the 100 stimuli employed. To ensure approximately 30 participants rated each stimulus, we opted to collect data from more participants than in our other studies (see Procedure below). One hundred fifty-four undergraduates $\left(M_{\text {age }}=18.57, S D=0.84\right)$ completed this study for partial course credit. The majority of participants self-identified as White (80.52\%) and female (65.58\%). No participants were excluded from analysis.

Materials. Stimuli consisted of digital photographs (i.e., mug shots) of 100 White men who were convicted murderers in the Florida State prison system as of October, 2014 (Wilson \& 
Rule, 2015). As with the CFD, attractiveness did not correlate with fWHR in the population of 452 faces we sampled from, $r(450)=-0.04, p=.396$. We selected the 50 prisoners who had the lowest fWHR $(M=1.66, S D=0.05)$ and the 50 prisoners who had the highest fWHR $(M=2.28$, $S D=0.08)$ from the larger database. This created two groups that significantly differed on fWHR, $t(98)=46.48, p<.001, d=9.39$, but not on attractiveness, $t(98)=0.56, p=.577, d=$ 0.11. All images were grayscaled and resized to a height of 354 pixels. Width varied across the images, but averaged 286 pixels.

Procedure. Because of the large number of stimuli (i.e., 100 stimuli in Study 3b) relative to our previous studies (i.e., 20 stimuli in Studies 1a, 1b, 1c, 1d, 2, and 3a) and experiment time restrictions, participants did not rate all 100 of the stimuli on all 12 infrahumanization items. Instead, five waves of participants (comprised of approximately 30 participants per wave) viewed a randomly selected subset of 20 of the possible 100 images. Participants always viewed 10 high fWHR faces and 10 low fWHR faces. Other than participants rating only a subset of the available faces, this study was procedurally similar to Study 3a. The only differences were that participants saw new stimuli (i.e., targets from the prisoner database) and that each target was displayed for 1 second instead of 500 milliseconds (a change due to software limitations).

\section{Results}

Of primary interest in the current study was whether high fWHR faces were infrahumanized relative to low fWHR faces. Emotion was effect coded (primary $=-0.5$, secondary $=0.5)$. There was a significant effect of emotion type, $B=-0.18, S E=0.03,95 \%$ CI [$0.24,-0.13], t(64.52)=-6.76, p<.001, d=1.68$. All targets were seen as more capable of primary $(M=4.84, S D=0.77)$ than secondary emotions $(M=4.66, S D=0.83)$, consistent with overall infrahumanization of prisoners. There was not a significant main effect of fWHR, $B=-$ 
$0.02 S E=0.05,95 \%$ CI $[-0.12,0.07], t(445.28)=-0.44, p=.663, d=-0.04$. Faces high $(M=$ $4.76, S D=0.79)$ and low in fWHR $(M=4.74, S D=0.80)$ were ascribed equivalent levels of emotions. However, we did observe the predicted interaction between fWHR and emotion type, $B=-0.10, S E=0.03,95 \%$ CI $[-0.16,-0.04], t(5872.79)=3.15, p=.002, d=-0.08$ (see Figure 4). To decompose this interaction, we looked at the effect of emotion type separately for high and low fWHR targets. Participants believed that low fWHR targets had less capacity to experience secondary emotions $(M=4.69, S D=0.83)$ than primary emotions $(M=4.82, S D=0.78), B=-$ $0.13, S E=0.02,95 \%$ CI $[-0.18,-0.09], t(139.16)=-5.92, p<.657, d=-1.00$. Similarly, participants also believed that high fWHR targets had less capacity to experience secondary emotions $(M=4.63, S D=0.85)$ than primary emotions $(M=4.86, S D=0.77), B=-0.23, S E=$ $0.03,95 \%$ CI $[-0.29,-0.18], t(143.14)=-8.56, p<.017, d=-1.43$. However, as is made clear by the significant interaction, this comparison was larger among the high fWHR targets than the low fWHR targets.

\section{Discussion}

Study $3 b$ provides an additional demonstration that fWHR influences infrahumanization. Replicating Study 3a, high fWHR targets were seen as less capable of experiencing secondary emotions than primary emotions. Unlike Study 3a, in Study 3b, low fWHR targets were also seen as less capable of experiencing secondary emotions than primary emotions. Importantly, although both the low and high fWHR targets were seen as more capable of experiencing primary compared to secondary emotions, this effect was larger for high fWHR targets. Because of their prison jumpsuits, it was readily apparent that the Study $3 \mathrm{~b}$ targets were prisoners, a chronically dehumanized group. Thus, according to infrahumanization theory, the targets should be denied secondary emotions relative to primary emotions. Yet, even though both high and low 
fWHR prisoners may be subjected to infrahumanization, these infrahumanizing judgments were stronger for the high fWHR than for the low fWHR targets.

\section{Study 4}

In Study 4, we investigated whether fWHR predicted humanness using another measure of humanity: Haslam's (2006) well-known senses of humanness. Specifically, Haslam makes a distinction between characteristics that are uniquely human (i.e., those that separate humans from other animals, such as morality), and characteristics that are associated with human nature (i.e., capacities that define the essence of humanity, such as curiosity). Denying someone human uniqueness likens them to animals (i.e., animalistic dehumanization) whereas denying someone human nature likens them to automata (i.e., mechanistic dehumanization).

To the extent that the association between high fWHR and aggression and dominance is indicative of animalistic dehumanization (e.g., high fWHR targets are animalistic, and therefore aggressive), we should observe that high fWHR targets would be denied human uniqueness. We were agnostic to the outcome of human nature characteristics. If high fWHR is a titrated signal of an animal-like nature, it might not influence ascriptions of human nature traits. However, it also seemed plausible that high fWHR targets would be denied humanity more broadly, leading to lower ascriptions of humanness on both dimensions.

\section{Method}

Participants. Fifty-one participants $\left(M_{\text {age }}=37.96, S D=13.96\right)$ completed Study 4 via Amazon's Mechanical Turk (mTurk) and were remunerated $\$ 0.40$ for their participation. Most participants identified as White (78.4\%) and female (56.9\%). No participants were excluded from analysis. 
Materials and Procedure. The materials and procedure for the current study were identical to Study 1a except as noted. After seeing each face, participants responded to eight items selected from Haslam and Bain's (2007) measure of dehumanization. Four items assessed perceived uniquely human characteristics (i.e., this person is broadminded, this person is conscientious, this person is humble, this person is polite; $\alpha=.90)$ and four items assessed perceived human nature characteristics (i.e., this person is active, this person is curious, this person is friendly, this person is helpful; $\alpha=.92$ ). Participants responded to each item on a 7point scale ranging from 1 (strongly disagree) to 7 (strongly agree). For the sake of experimental timing, we used the first four positively valenced items that loaded uniquely on the human uniqueness and human nature subscales respectively, rather than the entire scale. Finally, participants provided demographic information (e.g., sex, age, race), were debriefed, and were provided their compensation code. In our data, the human nature and human uniqueness subscales were positively correlated, $r(49)=.92, p<.001$.

\section{Results}

Of primary interest was whether fWHR biased the ascription of uniquely human and human nature characteristics. We observed a significant effect of sense of humanness such that all faces were ascribed more human nature traits $(M=4.32, S D=0.70)$ than uniquely human traits $(M=4.17, S D=0.67), B=0.14, S E=0.05,95 \% \mathrm{CI}[0.05,0.24], t(19.52)=3.19, p=.005$, $d=1.44$. We did not observe a significant main effect of fWHR, $B=-0.25, S E=0.21,95 \%$ CI [$0.71,0.19], t(14.84)=-1.23, p=.238, d=-0.64$. Faces with relatively high $\mathrm{fWHR}(M=4.11, S D$ $=0.75)$ and low fWHR $(M=4.37, S D=0.66)$ were not attributed differential levels of humanizing characteristics. Finally, the interaction between fWHR and sense of humanness was 
not significant, $B=-0.06, S E=0.08,95 \%$ CI $[-0.22,0.10], t(1974.925)=-0.70, p=.485, d=-$ 0.03 .

\section{Discussion}

Counter to predictions, in Study 4, we observed neither a main effect of fWHR nor an interaction between fWHR and sense of humanness. We did observe a main effect of sense of humanness such that all faces were ascribed more human nature traits than uniquely human traits, an effect consistent with past work (e.g., Haslam, Bain, Douge, Lee, \& Bastien, 2005). It may be the case that our abridged version of Haslam's (2006) senses of humanness measure hindered our ability to detect an effect, or that the effect was smaller when measured using this scale than anticipated. Nevertheless, although the predicted effect was not statistically significant, it was descriptively in the expected direction.

\section{Study 5}

The Studies thus far have provided converging evidence for the hypothesis that high fWHR faces are dehumanized relative to their low fWHR counterparts (albeit not across all measures; see Study 4). In these studies, we employed faces obtained from two distinct samples of stimuli (i.e., Chicago Face Database, Florida prisoners). Despite the advantages of these stimuli, our manipulation of fWHR was a naturalistic one - we were not randomly assigning faces to fWHR, but were relying on naturally occurring variations in fWHR. To provide a more robust test of the hypothesis that differences in fWHR lead to differences in perceived humanity we employed a new set of faces as stimuli in Study 5. Here, we used faces that were digitally manipulated to have both high and low fWHR versions (Stirrat \& Perrett, 2010). Of interest is whether the high fWHR version and the low fWHR version of the identical face identity are differentially dehumanized. 


\section{Method}

Participants. Because Study 5 used a within-target manipulation of fWHR, and a forcedchoice measure, it was possible that we would observe a smaller effect. Thus, we conservatively opted to collected data from at least 200 participants. In actuality, 231 mTurk workers $\left(M_{\text {age }}=\right.$ 34.32, $S D=11.36)$ completed this study for $\$ 0.30$. Most participants identified as White $(69.0 \%)$ and female $(52.8 \%)$.

Materials and Procedure. We used 12 White male targets that were digitally manipulated so as to yield high and low fWHR versions of each (for stimulus creation, see Stirrat \& Perrett, 2010), netting 24 total stimuli (i.e., 12 pairs of high/low fWHR targets). We adapted the instructions from Study 1a for use in Study 5. Specifically, participants were told "People can vary in how human-like they seem. Some people seem highly evolved whereas others seem no different from lower animals. In this survey, you will be shown pairs of faces of nearly identical faces. You will be asked to indicate which of the two seems more human. Simply click on the face you believe is more human." Participants were shown the Ascent of Man image on the instruction screen, and were told to "rely on their gut instinct" when making responses. Participants completed 12 trials. On each trial, participants were shown both the high and low fWHR version of one face identity. They then chose which of the two faces seemed more human. Presentation order was independently randomized for each participant. After completing all 12 trials, participants were asked to provide demographic information, were debriefed, and were provided their completion code to receive compensation.

\section{Results}

Of primary interest was the extent to which participants preferentially selected the high or low fWHR face as seeming more human. Counts of high and low fWHR selections were 
submitted to a mixed-model analysis. We observed a marginally significant effect of fWHR, $B=$ $-0.09, S E=0.05,95 \%$ CI $[-0.21,0.02], t(18.67)=-1.75, p=.096, d=-0.81$. Converting the counts to proportions reveals that high fWHR faces $(M=0.45, S D=0.22)$ were selected marginally less often than low fWHR faces $(M=0.54, S D=0.22)$ when asked to choose which face in the set seemed more human.

\section{Discussion}

The results of Study 5 provide marginally significant evidence suggesting that high fWHR targets are dehumanized relative to low fWHR targets, even when face identity is held constant. Here, we took naturally occurring faces and digitally manipulated them so as to create high and low fWHR versions of the same face identity. Using a face-valid measure of humanity (i.e., participants were asked which of two simultaneously presented faces is more human), we observed that the high fWHR version of the same face identity tended to be seen as less human than the low fWHR version. However, given the marginal nature of the observed effects, these inferences must be made with some caution.

\section{Study 1-5 Summary}

Our goal across Studies 1-5 was to test whether facial width-to-height ratio biases perceptions of humanness across a variety of commonly used operationalizations of humanness and across multiple target and stimulus dimensions. Indeed, in these initial studies, we showed that high fWHR targets are blatantly dehumanized (Studies 1a, 1b, and 1d) are seen as having minds that can feel sensations, but not engage in sophisticated cogitation (Study 2), are infrahumanized and perceived as incapable of feeling sophisticated secondary emotions (Studies $3 a$ and $3 b$ ), and tend to be judged as less human even while holding identity constant (Study 5). It is worth noting that two studies (i.e., Study 1c and Study 4) did not produce statistically 
significant effects consistent with hypotheses and one study (i.e., Study 5) produced marginally significant effects; nevertheless, the non-significant trends were descriptively in the predicted direction. To summarize the effect of fWHR on measures of dehumanization, we conducted a small meta-analysis (Goh et al., 2016) on the effects observed across all samples included in Studies 1-5. This analysis suggests that our observed effects of fWHR across multiple measures of dehumanization are reliable, $r=0.28,95 \%$ CI $[0.21,0.35], z=7.10, p<.001$.

These findings are not readily attributed to a general valence halo effect. First, at the level of the face stimuli, we matched the high and low fWHR faces on physical attractiveness in most studies, a key signal of valence. Admittedly, for studies using stimuli from the Chicago Face Database, we selected a relatively small number of stimuli. Therefore, the between-group effects are statistically underpowered, rendering it difficult to accept the null that our fWHR groups do not differ based on the inferential test alone. However, little evidence exists that fWHR correlates with attractiveness in the populations from which we obtained our stimuli. Further, at the level of the measures, we employed stimuli that differed in valence. Specifically, in Studies $3 a$ and $3 b$, which used the infrahumanization measure of primary and secondary emotions, we employed both positive and negative emotions, as is typical in this literature. Thus, we had both positive and negative primary and secondary emotions, and the effect of fWHR on infrahumanization still obtained.

\section{Ancillary Studies: Investigating a fWHR-humanness mechanism}

Although Studies 1-5 provide evidence suggesting that high fWHR individuals are ascribed less sophisticated faculties, compared to low fWHR individuals, they do not provide evidence for why this occurs. Here, we considered two possibilities. One possibility is that the fWHR-humanness link is mediated by inferences of fWHR-linked traits. As summarized above, 
fWHR has been linked to a variety of traits that may be pertinent to dehumanization, such as the perception that high fWHR targets are more impulsively aggressive, angry, and dominant. Thus, the tendency to see high fWHR targets as lacking human sophistication may be driven by such trait inferences. Alternatively, it is possible that the fWHR-humanness link may be a "direct" one. Perhaps the tendency to dehumanize high fWHR targets is so culturally over-learned (or, perhaps high fWHR targets are seen as occupying dehumanized roles, such as prisoner) that the fWHR-humanness link need not occur via perceptions of targets impulsive aggressiveness and the like.

To better understand this process, we conducted two Ancillary Studies in which we explore a range of possible traits extracted from the fWHR literature as potential mediators of the fWHR-humanness link. Ancillary Study 1 involves a re-analysis of existing data (both from Studies 1a-1d and from the Chicago Face Database), whereas Ancillary Study 2 involves the collection of new data (including a conceptual replication of Study 1a). Of interest in both studies is whether traits that have been previously linked to fWHR (e.g., dominance) may mediate the fWHR-to-humanness link observed above.

\section{Ancillary Study 1}

In the first Ancillary Study, we conducted a re-analysis of the fWHR-humanness link for the White male faces in our Study 1 series of experiments (i.e., Studies 1a, 1b, 1c, \& 1d), investigating the possibility of potential mediators for the observed effects. We focused on these data in particular for multiple reasons. First, blatant dehumanization is the most face valid measure of humanness that we employed in our studies above. Second, by combining the data for the White male faces across these four studies, we have a relatively large number of participants (201 participants). Third, because the Chicago Face Database has pre-tested each of 
these faces on a variety of theoretically relevant traits (including anger, dominance, masculinity, threat, and trustworthiness), this allows us to use existing, normed data to conduct the mediation analyses.

\section{Method}

In this first Ancillary Study, we tested the extent to which perceived anger, dominance, masculinity, threat, and trustworthiness mediated the fWHR-humanness effect. Values for perceived anger, dominance, masculinity, threat, and trustworthiness were obtained from the Chicago Face Database (Ma et al., 2015). All mediators were rated on scales ranging from 1 (not at all) to 7 (extremely).

\section{Results}

Each analysis was run separately in a mixed-model framework. To test mediation in this framework, we used the Monte Carlo method (MacKinnon, Lockwood, \& Williams, 2004). For each analysis, we used 20,000 bootstrapped samples to test for a significant indirect effect. Results revealed that perceived dominance, 95\% CI [1.01, 2.74], masculinity, 95\% CI [1.17, 2.43], and trustworthiness, 95\% CI [-1.26, -0.69], were all significant mediators. Perceived anger, 95\% CI [-1.45, 0.17], and perceived threat, 95\% CI [-1.84, 0.15], were not.

\section{Discussion}

Results from this first Ancillary Study support the notion that the influence of fWHR on dehumanization occurs indirectly via trait inferences driven by variations in fWHR. Specifically, this analysis provides evidence that perceived dominance, trustworthiness, and masculinity mediate the fWHR-to-humanness link.

Although this re-analysis of the existing data has a variety of benefits, it does rely on a relatively limited set of stimuli (a limitation true of most of our studies above). Thus, we 
conducted a second Ancillary Study designed to investigate potential fWHR-to-trait links established in the literature as potential mediators for the fWHR-to-humanness link we have observed, but using a much larger sample of target stimuli.

\section{Ancillary Study 2}

The goal of the second Ancillary Study was to provide a more robust understanding of the mechanisms that undergird the fWHR-to-humanness association. Further, by using the blatant dehumanization measure from Study 1, this also allowed for a replication of the fWHRhumanness link using a larger set of stimuli.

\section{Method}

Participants. Forty-three participants $\left(M_{a g e}=33.12, S D=9.71\right)$ completed this Ancillary Study via Amazon's Mechanical Turk, and were remunerated \$0.50 for their participation. Most participants identified as White $(76.7 \%)$ and male (51.2\%). No participants were excluded from analysis.

Procedure. The procedure was identical to Study 1b, except as noted. Participants rated 72 faces (36 White male; 36 White female) from the Chicago Face Database using the blatant dehumanization scale.

\section{Results}

In this Ancillary Study, we used a fuller range of stimuli that did not merely represent the extremes of the fWHR distribution. Therefore, fWHR was treated continuously in these analyses. Because we had new participants making new ratings, we first tested whether, in this sample, fWHR predicted dehumanization. Replicating the effects presented above, fWHR predicted blatant dehumanization, $B=-13.37, S E=2.85,95 \%$ CI $[-19.12,-7.61], t(42)=-4.69, p<.001, d$ $=-1.45$. 
Next, paralleling the mediation analyses reported in Ancillary Study 1, we tested the extent to which perceived anger, dominance, masculinity, threat, and trustworthiness mediated the fWHR-humanness effect. Results revealed that perceived anger, 95\% CI [-3.03, -0.97], dominance, $95 \%$ CI [-3.15, -0.74], and threat, 95\% CI [-4.18, -1.80], were significant mediators. Perceived masculinity, 95\% CI [-0.78, 0.69], and trustworthiness, 95\% CI [-1.54, 0.85], were not.

\section{Discussion}

As outlined in the introduction, facial width-to-height ratio has effects on a constellation of face-based trait evaluations and judgments. These Ancillary Studies indicate that these traits, in turn, can predict the extent to which another person is blatantly dehumanized. Although the traits that served as significant mediators did differ somewhat across the two studies (e.g., anger was a mediator in Ancillary Study 2, but not Ancillary Study 1), perceived dominance was a significant mediator in both Studies. This makes sense given that dominance is central to most of the existing literature on fWHR-trait links.

Although each mediator that we tested was theoretically motivated by existing effects in the literature, we stress that these findings are exploratory in nature - we did not have strong a priori predictions about which indirect effects would be significant. Further, caution is warranted because mediation tests cannot definitively prove causal relationships (e.g., Fiedler, Schott, \& Meiser, 2011), nor can they distinguish between whether dominance (for example) mediates humanness effects, or whether humanness effects mediate dominance judgments - both such models are in the same equivalence class, statistically speaking (Thoemmes, 2015). However, these Ancillary Studies offer the beginnings of an understanding about how dominance related traits may play an important role in the fWHR-humanness link. 
In the final three studies, we tested the extent to which the fWHR-humanness link has implications that extend beyond well-established operational definitions of humanness or mind ascription. Specifically, we sought to test whether this this link has implications for judgments about criminality (Study 6), perceived utility in social roles (Study 7), and broader success in valued life domains (Study 8).

\section{Studies 6-8: Investigating downstream consequences of a fWHR-humanness link}

\section{Study 6}

In the previous studies, we investigated the possibility of a fWHR-humanness link, and the extent to which that link could be accounted for, statistically speaking, by established fWHRtrait links (e.g., dominance). In the latter three studies, we investigate the possibility that this “upstream" fWHR-humanness link may influence social judgment beyond perceptions of targets' faculties. Specifically, in the latter three studies, we investigate whether fWHR influences “downstream" social judgments, such as fits to social roles, and professional and life success.

In Study 6, we focused a domain that is consensually negative: criminality. Specifically, participants judged the likelihood that criminals were imprisoned for committing an unsophisticated crime (i.e., assault) or for committing a crime that ostensibly requires sophistication and planning (i.e., embezzlement). We tested two competing hypotheses for Study 6 - a stereotype fit hypothesis consistent with dehumanization and a halo effect hypothesis consistent with prejudice (i.e., negative valence). The stereotype fit hypothesis predicts that high and low fWHR targets should be seen as differential fits to these crimes because they are differentially capable of engaging in sophisticated and simplistic behaviors (see Bodenhausen \& Wyer, 1985; Macrae \& Shepard, 1989). Alternately, if this is a negativity halo effect (i.e., high 
fWHR targets are merely disliked), high fWHR targets should be seen as more likely to commit any crime relative to low fWHR targets because they are derogated overall.

\section{Method}

Participants. Forty-eight mTurk workers completed this study and were compensated $\$ 0.50$ for their participation. This sample $\left(M_{\text {age }}=37.67, S D=12.88\right)$ was primarily White (79.2\%) and female $(52.1 \%)$.

Materials. The stimuli in this experiment were a subset of the stimuli used in Study $3 b$. Specifically, we showed participants 40 images of convicted murderers, half of which were the 20 lowest fWHR targets in the Florida State criminal face database and the other half of which were the 20 highest fWHR targets in the database (Wilson \& Rule, 2015).

Procedure. After providing informed consent, participants were told that people are surprisingly good at gleaning personality information from faces, even when presented briefly, and that we were interested if this effect extended to knowledge about what sort of crime a prisoner committed. Participants then viewed 40 faces, one at a time. Presentation order was independently randomized for each participant and faces were displayed onscreen for $500 \mathrm{~ms}$. Participants were asked to separately rate how likely it was that each individual was in prison for assault and for embezzlement. Participants responded to both items for each target on 7-point scales ranging from 1 (very unlikely) to 7 (very likely). Participants then provided demographic information, were debriefed, and were provided their completion code to receive compensation.

\section{Results}

Of primary interest was the extent to which targets with high and low fWHR were perceived to be guilty of different crimes. Crime type was effect coded (assault $=-0.5$, embezzlement $=0.5)$. We observed a main effect of crime type, $B=-0.65, S E=0.13,95 \%$ CI $[-$ 
$0.90,-0.40], t(45.15)=-5.18, p<.001, d=-1.54$, reflecting that overall, targets were perceived as more likely to have committed assault $(M=4.75, S D=0.72)$ than embezzlement $(M=4.10$, $S D=0.78)$. This analysis did not yield the main effect of fWHR predicted by a halo effect, $B=$ $0.01, S E=0.05,95 \%$ CI $[-0.09,0.11], t(4086.67)=0.27, p=.791, d=0.01$. Instead, it yielded an interaction of fWHR and crime type predicted by the fit hypothesis, $B=-1.49, S E=0.19,95 \%$ CI [-1.87, -1.11$], t(46.85)=-7.97, p<.001, d=-2.33$ (see Figure 5). We decomposed this interaction by comparing the effect of fWHR separately for assault and embezzlement. High fWHR targets $(M=5.13, S D=0.81)$ were perceived as more likely to be in prison for assault than low fWHR targets $(M=4.37, S D=0.79), B=-0.76, S E=0.28,95 \%$ CI $[0.19,1.33]$, $t(42.25)=2.71, p=.010, d=0.83$. However, as predicted by the stereotype fit hypothesis, perceivers believed that high fWHR targets $(M=3.73, S D=0.94)$ were less likely to be in prison for embezzlement than low fWHR targets $(M=4.46, S D=0.76), B=-0.74, S E=0.26,95 \% \mathrm{CI}$ $[-1.27,-0.21], t(42.90)=-2.83, p=.007, d=-0.86$.

\section{Discussion}

Study 6 provides evidence that the fWHR-humanness link has downstream implications for perceptions of criminality. We did not observe the main effect of fWHR predicted by the halo effect hypothesis, suggesting that these judgments are not merely the result of prejudice directed at high fWHR individuals. Instead, and consistent with the stereotype fit hypothesis, high fWHR targets were seen as more likely to be in prison for a relatively unsophisticated crime (i.e., assault) than low fWHR targets whereas low fWHR targets were seen as more likely to be in prison for a relatively sophisticated crime (i.e., embezzlement). The stereotype fit between crime and the sophistication or simplicity signaled by fWHR appears sufficient to elicit the 
downstream judgment effects. Whereas embezzlement may require the planning of a calculating intellect, assault requires nothing more than a fist and an impulse.

\section{Study 7}

Results to this point suggest that relatively high fWHR people are routinely denied sophisticated human capacities and are condemned as unsophisticated criminals. However, in Study 6, we provided evidence that this was not merely the consequence a negativity halo. Instead, results suggested that high fWHR targets are not simply seen as generally bad or incapable of everything; rather, they are seen as incapable specifically of sophisticated thoughts and actions. Carrying this logic forward, it may be true that in some situations, high fWHR targets should be preferred. Consistent with this hypothesis, work by Hehman and colleagues (2015) showed that participants preferred higher fWHR individuals when choosing partners in a competitive context. Specifically, in their first study, participants were shown an array of digitally generated faces that varied in their fWHR and were asked to select who would be on their own and the opposing team in a competitive context. Participants tended to select the high fWHR faces for their own team, relegating the low fWHR individuals to the opposition. In follow-up studies, high fWHR targets were selected at a rate greater than chance for boxing and football teams, but less than chance for a chess team.

We designed the current study as a conceptual replication of the work of Hehman and colleagues (2015) using different stimuli, methods, and measures to test whether there may be times in which high fWHR targets are favored compared to low fWHR targets. Mirroring the stereotype fit logic of Study 6 (and the contravening negativity halo hypothesis), we predicted that high fWHR targets would be seen as an especially strong fit to simple, physical tasks but not sophisticated, mental tasks. 


\section{Method}

Participants. We recruited 40 mTurk workers $\left(M_{\text {age }}=36.40, S D=12.58\right)$ for this study and compensated them $\$ 0.50$ for their participation. The majority of our participants identified as White $(77.5 \%)$ and as male $(60.0 \%)$.

Materials and Procedure. The materials and procedure for the current study were identical to Study 1a except as noted. After seeing each face, participants responded to eight items designed to assess how helpful the target would be in a variety of situations. Four items measured situations requiring physicality (i.e., backing you up in a fight, replacing the shingles on a house, moving heavy furniture, playing on a competitive hockey team; $\alpha=.88$ ) and four items measured situations requiring mental sophistication (i.e., filling out a complex tax return, solving a challenging puzzle, planning investments, discussing the opera; $\alpha=.92)$. Participants responded to each item on a 7-point scale ranging from 1 (strongly disagree) to 7 (strongly agree).

\section{Results}

Of primary interest was the relationship between fWHR and perceived helpfulness. Activity type was effect coded $(-0.5=$ mental, $0.5=$ physical $)$. There was a significant main effect of activity type, $B=0.87, S E=0.12,95 \% \mathrm{CI}[0.63,1.11], t(39)=7.25, p<.001, d=2.32$. All targets were seen as more helpful for physical $(M=4.12, S D=0.79)$ than mental $(M=3.13$, $S D=0.80)$ tasks. Inconsistent with a halo effect hypothesis, we did not observe a significant main effect of fWHR, $B=0.19, S E=0.13,95 \%$ CI $[-0.07,0.46], t(18.99)=1.51, p=.148, d=$ 0.69. High fWHR targets $(M=3.72, S D=0.70)$ were seen as equally helpful across all domains as low fWHR targets $(M=3.54, S D=0.75)$. Finally, consistent with a stereotype fit hypothesis, we observed an interaction between fWHR and activity type, $B=1.15, S E=0.15,95 \% \mathrm{CI}[0.84$, 
1.45], $t(39)=7.71, p<.001, d=2.47$ (see Figure 6). We decomposed this interaction by comparing the effect of fWHR separately for physical and mental tasks. High fWHR targets were seen as more helpful for tasks requiring physicality $(M=4.50, S D=0.91)$ than for tasks requiring mental acumen $(M=3.06, S D=0.91), B=1.45, S E=0.17,95 \%$ CI $[1.09,1.80], t(39)$ $=8.32, p<.001, d=2.67$. Low fWHR targets were also seen as more helpful for tasks requiring physicality $(M=3.74, S D=0.85)$ than for tasks requiring intellect $(M=3.44, S D=0.93), B=$ $0.30, S E=0.10,95 \%$ CI $[0.10,0.50], t(39)=3.03, p=.004, d=0.97$. However, as indicated by the significant interaction, this effect was smaller for low fWHR than for high fWHR targets.

\section{Discussion}

Study 7 conceptually replicates the work of Hehman and colleagues (2015), providing converging evidence for the hypothesis that in certain circumstances, high fWHR individuals are preferred relative to their low fWHR counterparts. Although participants viewed both high and low fWHR targets as more helpful in physical than in mental situations (perhaps because moving furniture is easier than doing taxes), this difference was much larger for high fWHR targets. In other words, whereas participants saw high fWHR targets as substantially more helpful for physical tasks than for mental tasks, participants did not as strongly distinguish between task type when judging how helpful they thought low fWHR targets would be. Importantly, these findings suggest that perceivers do not uniformly derogate high fWHR targets. Rather, high and low fWHR targets are each seen as being good fits to different roles. Although perceivers especially valued high fWHR targets for activities requiring physicality, facial width-to-height ratio is independent of body size (Weston et al., 2007) and, to the authors' knowledge, no research has linked fWHR with intelligence. Therefore, it is unlikely that fWHR is being used as an honest signal of physical size or of intelligence. Rather, we have argued, fWHR is signaling 
the extent to which someone has complex or simple faculties or inner states. However, it is also worth noting that some of the tasks chosen for the dependent measure may have been stereotypically masculine physical domains (e.g., fighting and moving furniture). Given the overlap between fWHR and physical dominance, this may play a role in participants' responses. Thus, an open question is the extent to which these or similar effects would emerge in stereotypically female domains, or with female faces that vary in fWHR (as in Study 1b).

\section{Study 8}

Whereas Studies 6 and 7 investigate how high versus low fWHR targets are seen as differential fits to roles (both positive and negative), in Study 8 we sought to investigate whether the dehumanization of high fWHR targets led them to be seen as deficient in domains consensually valued to humans, such as family life, relationships, work, and more generally, whether this influenced perceptions of life satisfaction. Dimensions such as family, relationships and work are central to nearly all major models of human life satisfaction and happiness, in part because success in these domains often accounts for a great deal of the variance in whether a person is leading a satisfying and fulfilling life (e.g., Iverson \& Maguire, 2000; Pavot \& Diener, 2008; Veenhoven, 1996). Of interest was whether high fWHR targets would be seen not just as less-than-fully-human, but as failing in valued life domains.

To investigate this, participants rated high and low fWHR targets on four life success items adapted from Dion and colleagues' (1972) facial attractiveness research: romantic success, parental success, work success, and personal fulfillment. Participants also rated these targets' subjective happiness (Lyubomirsky \& Lepper, 1999). Of interest was whether, relative to low fWHR targets, high fWHR targets would be perceived as unsuccessful and unsatisfied in life.

\section{Method}


Participants. Fifty mTurk workers $\left(M_{\text {age }}=35.98, S D=12.66\right)$ completed this study and were remunerated $\$ 0.50$ for their participation. The majority of our sample identified as White $(80.0 \%)$ and as female $(54.0 \%)$.

Materials and Procedure. The materials and procedure for the current study were identical to Study 1a except as noted. After seeing each face, participants completed a 4-item life success scale adapted from Dion and colleagues (1972) which asked participants to estimate how likely they thought each target would have "romantic success," "career success," "parental success," and "achieve personal fulfillment," on 7-point scales ranging from 1 (strongly disagree) to 7 (strongly agree). Participants also completed Lyubomirsky and Lepper's (1999) four-item subjective happiness questionnaire for each face, adapted such that responses were about the target rather than about the self (e.g., In general, this person is a happy person), using 7-point scales ranging from 1 (strongly disagree) to 7 (strongly agree).

\section{Results}

We first created composite measures for the life success items $(\alpha=.96)$ and the perceived happiness items $(\alpha=.78)$ separately for high and low fWHR targets. Because these two measures were highly correlated $(r=0.72)$ we combined them into a single measure of global life success. ${ }^{5}$ Counter to predictions, we did not observe a significant effect of fWHR, $B=$ $-0.16, S E=0.25,95 \%$ CI $[-0.68,0.37], t(18.07)=-0.63, p=.534, d=-0.30$. High fWHR targets $(M=3.63, S D=0.58)$ were rated equivalently positively compared to their low fWHR counterparts $(M=3.79, S D=0.61)$.

\section{Discussion}

${ }^{5}$ Analyzing these measures independently yields nearly identical results (life success: $p=.510$; perceived happiness: $p=.564$ ). 
Contrary to hypotheses, Study 8 did not provide evidence that targets' fWHR influenced blanket perceptions of targets' life outcomes. On a combined measure of perceptions of success in roles commonly valued by humans and overall well-being, high and low fWHR targets were rated as having equivalent outcomes.

Taken in the context of the previous studies, and especially of Study 7, this seems to indicate that the downstream consequences of fWHR on social judgment are nuanced. It is not simply that high fWHR targets are "bad" or are seen as failing at life; rather, high fWHR targets are seen as good fit to roles that may be psychologically simple but physically demanding. Conversely, it appears that low fWHR targets are not seen as "good" or as "winners;" rather, their faculties are seen as congruent with roles befitting their higher levels of perceived inner sophistication.

\section{General Discussion}

The current work tested the hypothesis that facial width-to-height ratio - a structure ubiquitous to human faces - biases perceptions of humanness. We find consistent evidence supporting this hypothesis. Compared to their low fWHR counterparts, high fWHR people were blatantly dehumanized (Studies 1a, 1b, 1d), were seen as having fewer agentic capacities (Study 2), were seen as less capable of experiencing uniquely human emotions (Studies 3a \& 3b), and tended to be seen as less human even when identity was held constant (Study 5). However, these results did not extend to perceptions of human nature and human uniqueness (Study 4). Further, two Ancillary Studies demonstrated that the effect of fWHR on perceptions of blatant dehumanization are mediated by a constellation of trait evaluations that are theoretically consistent with both the broader facial width-to-height ratio and dehumanization literatures. Whereas the two different procedures in these Ancillary Studies yielded somewhat different 
patterns of mediating traits, ratings of dominance were a consistent mediator of the fWHRhumanness link across the studies. Finally, we also provided evidence suggesting that the dehumanizing perceptions of high fWHR individuals have consequences for downstream social judgments. Compared to low fWHR individuals, participants estimated that high fWHR individuals are more likely to be in prison for an unsophisticated crime like assault, but not a sophisticated crime such as embezzlement (Study 6), and deemed them more suitable for physical, but not mental tasks (Study 7). However, these results did not extend to high fWHR targets being seen as being bad or failing at life more generally (Study 8). These results provide consistent evidence that facial width-to-height ratio serves as a cue for humanness, broadly construed.

Meta-Analysis. Despite the consistency of the fWHR-humanness link across multiple target populations, samples, measures, and methods, three studies failed to obtained statistical significance (Studies 1c, 4, \& 8) and one study was marginally significant (Study 5). To provide greater understanding of the reliability of the fWHR-humanness link, we conducted a metaanalysis across all of the measures of humanness collected across all of the studies (Goh et al., 2016). To do this, we computed effect sizes for the effect of fWHR on blatant dehumanization in Studies 1a, 1b, 1c, 1d, \& Ancillary Study 2, the effect of fWHR on sense of humanness in Study 4, the effect of fWHR on humanness in Study 5, the effect of fWHR on global life success in Study 8, the interactive effect of fWHR and mind dimension in Study 2, the interactive effect of fWHR and emotion type in Studies 3a and 3b, the interactive effect of fWHR and crime type in Study 6, and the interactive effect of fWHR and activity type in Study 7, yielding a total of 13 effects across 916 total participants. 
All effect sizes were weighted by sample size. As shown in Figure 7, this analysis yields a statistically significant meta-analytic effect, $r=0.35,95 \%$ CI $[0.30,0.41], z=10.10, p<.001$. The results of this meta-analysis provide cumulative evidence of the effect of fWHR on ascriptions of humanity to others, suggesting that the effect is reliable and of medium magnitude by Cohen's conventions.

We see this work as important for a number of reasons. Although existing evidence suggests that both group and individual motives influence the perception of others' humanity, relatively little work has explored bottom-up determinants of dehumanization (cf., Hugenberg et al., 2016; Schroeder \& Epley, 2015). The current work suggests that bottom-up cues in others' faces have consequences for when we ascribe and withhold minds. Additionally, this work has implications for several literatures, including dehumanization, face perception, and criminal justice, which we outline below.

Dehumanization. The current work has meaningful implications for research exploring when, why, and to whom we ascribe and deny sophisticated cognitive and emotional faculties. Although we acknowledge real differences between literatures on infrahumanization, dehumanization, and mind perception, in the current work we have highlighted one domain in which many of these models have similar effects. This work was originally designed to test the extent to which fWHR-based dehumanization occurs across a variety of measures. Yet, by using multiple well-established measures, we may be able to triangulate on the specific type of dehumanization to which high fWHR faces are subjected. Although high fWHR targets are seen as lacking in agency (Study 2), they are still seen as highly capable of assault and physical actions (Studies 6 and 7). These are behaviors that require some ability to act upon the world but are not actions that require planning and sophistication. Further, the blatant dehumanization 
measure (Kteily et al., 2015) correlates robustly with perceptions of unsophistication and savagery. Together, these results suggest that high fWHR targets are perceived as lacking the ability for intentional action, self-regulation, and complex emotion, and yet still capable of (and even preferred for) basic physical experiences and tasks.

Another advantage of the current work is that these or similar methods could be leveraged in the future with an eye toward theoretical integration (see Bain et al., 2014). Few studies rely on multiple measures of human faculties originating from different theoretical perspectives. Such work might be of benefit in future research, especially when attempting to understand how a specific group or characteristic might be targeted with dehumanizing responses. Given the conceptual relations between these different theoretical frameworks, we would be excited to see future research clarify where and why these models empirically converge and diverge.

Face Perception. The current work has implications for our understanding of how stable characteristics of faces influence person perception. Most past work exploring the link between faces and person perception has focused on how facial features (e.g., Montepare \& Zebrowitz, 1998; Zebrowitz, 1997; Zebrowitz \& Montepare, 1992, 2008) or facial similarity to emotional expressions (e.g., Oosterhof \& Todorov, 2008; Oosterhof \& Todorov, 2009; Todorov et al., 2008) predict ascriptions of traits. Very little work exists linking face perception with beliefs about others' humanity. What research does exist demonstrates that configural face processing a feature integration process unique to human faces - can trigger humanness in a bottom-up manner (e.g., Hugenberg et al., 2016; Fincher \& Tetlock, 2016), as can certain facial features that trigger configural processing (e.g., direct eye gaze, Khalid, Deska, \& Hugenberg, 2016; Young, Slepian, Wilson, \& Hugenberg, 2014). However, the current work shows that at least one 
specific facial structure inherent in all faces, facial width-to-height ratio, signals humanity without manipulating configural face processing.

As a feature of faces, fWHR has garnered a great deal of focus in recent years. The vast majority of research exploring fWHR has focused on the behavioral patterns of men, demonstrating a small, but reliable link between fWHR and behavioral indicators of aggression (Haselhuhn et al., 2015). In the current work, we focused on fWHR as a facial stereotype, demonstrating the relationship between fWHR and how targets are perceived. Past work has found that individuals with relatively higher fWHR are perceived as threatening, dominant, and angry (e.g., Geniole et al., 2015). Our work extends this literature by showing that these links between fWHR and trait judgments (such as dominance) have implications for how people perceive the inner life of others. Specifically, relatively high fWHR individuals are seen as possessing less humanlike minds and as better suited for mentally and emotionally unsophisticated than for mentally and emotionally sophisticated roles.

Criminal Justice. From Gray and colleagues' (2007) perspective, minds have two distinct dimensions, agency and experience. Those with agency are moral actors who are capable of being right and wrong whereas those with experience are moral patients who are capable of having right and wrong done unto them. We find that high fWHR individuals are denied agency but not experience, a pattern consistent with moral patiency. The vulnerability of moral patients suggests that they should be the most deserving of protection. Indeed, when criminal acts are committed against children or puppies (i.e., targets with a great deal of experience but little agency) backlash is fierce and recrimination is quick. However, a dearth of agency implies an impaired ability to know right from wrong. Indeed, children and canines are rarely considered actors fully capable of knowing right from wrong. Yet, an aggressive dog may be put to death. 
Here, the dog's fundamental inability to consider the moral consequences of its actions suggest that its behavior is incorrigible and that it is beyond redemption. Perhaps high fWHR individuals are not perceived as fully culpable for their criminal actions compared to low fWHR individuals, who presumably have the agentic capacity to know right from wrong, act with intention, and form premediated plans. Indeed, in order for an act to be considered legally criminal, mens reacriminal intent - must be established. And yet, perhaps precisely because of that lack of perceived agency, they may be seen as less capable of remediation and self-regulation in the future.

In another important link to criminal justice, we showed that participants believe a high fWHR individual is more likely to be in jail for assault than a low fWHR individual. In the United States, one is much more likely to receive a capital sentence for a violent crime than for a relatively complex, but non-violent crime such as embezzlement, suggesting that high fWHR individuals may be at increased risk for capital punishment. Indeed, recent work reified this exact hypothesis - fWHR independently predicted the likelihood that convicted murders received the death penalty (Wilson \& Rule, 2015).

\section{Limitations and Future Directions}

Despite the implications of the current findings, there are a number of limitations to the current work that we believe can provide avenues for future research. First, in most studies, we intentionally asked participants to make comparisons between faces that varied quite drastically in their fWHR, and we primarily used faces from the Chicago Face Database. This approach was intentional. We chose to focus primarily on extreme ends of the fWHR distribution to provide clear evidence for the existence of the fWHR-based dehumanization effect, and we used the CFD because it is one of the highest quality, rigorously controlled extant face databases. Yet, this 
strategy may have caused the size of our effects to be inflated. However, we did observe analogous effects with the faces of prisoners, showed preliminary evidence that the effects may generalize to White female and Black male targets, and found that an analogous effect occurred with a larger sample of CFD faces in which fWHR was treated as a continuous variable. Just as important, we also used cross-classified mixed-model analyses that treat both participant and stimulus as random effects - again limiting potential concern about stimulus effects. Nevertheless, future work would do well to present a fuller range of stimuli across the fWHR spectrum as well as test more robustly the extent to which these effects occur when using faces of individuals other than White men.

Additionally, although we believe the present research has consistently suggested that high fWHR faces are dehumanized, there are a number of important, yet unanswered, questions. For example, whereas we found that fWHR can influence dehumanization, and that fWHR can influence downstream social judgment in ways consistent with that dehumanization, we have not provided direct evidence that the fWHR-to-judgment relationship (i.e., the Studies 6-8 effects) are caused by dehumanization (i.e., the Studies 1-5 effects). Future research would benefit from directly demonstrating such a causal pathway. Similarly, the present research does not provide evidence for the origin of the fWHR-dehumanization link. Given that fWHR does appear to predict actual social behavior, one possibility for the origin of this link is a "kernel of truth" in differential behaviors of high and low fWHR individuals. However, this is by no means the only possibility, and future research would benefit from addressing the origin of the fWHRdehumanization link. Further, the present research does not demonstrate how the downstream consequences of the fWHR-dehumanization link play out in social interactions. One possibility is that treatment resulting from one's own fWHR could lead to either internalization (e.g., 
coming to believe one is ill suited to sophisticated intellectual labor) or that such treatment could elicit a self-fulfilling prophecy over time. This too could serve as an interesting area for future research.

\section{Conclusion}

The current work suggests that facial width-to-height ratio has meaningful consequences for person perception. Specifically, we provide evidence for the novel hypothesis that individuals with relatively high facial width-to-height ratio are routinely denied their full humanity. 


\section{References}

Alrajih, S., \& Ward, J. (2014). Increased facial width-to-height ratio and perceived dominance in the faces of the UK's leading business leaders. British Journal of Psychology, 105, 153161.

Anderl, C., Hahn, T., Schmidt, A. K., Moldenhauer, H., Notebaert, K., Clément, C. C., \& Windmann, S. (2016). Facial width-to-height ratio predicts psychopathic traits in males. Personality and Individual Differences, 88, 99-101.

Bain, P. G., Vaes, J., \& Leyens, J. P. (2014). Humanness and dehumanization. New York, NY: Taylor \& Francis.

Balas, B., \& Horski, J. (2012). You can take the eyes out of the doll, but... Perception, 41, 361364.

Balas, B., \& Tonsager, C. (2014). Face animacy is not all in the eyes: Evidence from contrast chimeras. Perception, 43, 355-367.

Bird, B. M., Jofré, V. S. C., Geniole, S. N., Welker, K. M., Zilioli, S., Maestripieri, D., ... \& Carré, J. M. (2016). Does the facial width-to-height ratio map onto variability in men's testosterone concentrations?. Evolution and Human Behavior, 37, 392-398.

Blair, I. V., Judd, C. M., \& Fallman, J. L. (2004). The automaticity of race and Afrocentric facial features in social judgments. Journal of Personality and Social Psychology, 87, 763-778.

Bodenhausen, G. V., \& Wyer, R. S. (1985). Effects of stereotypes in decision making and information-processing strategies. Journal of Personality and Social Psychology, 48, 267-282. 
Carré, J. M., \& McCormick, C. M. (2008). In your face: Facial metrics predict aggressive behaviour in the laboratory and in varsity and professional hockey players. Proceedings of the Royal Society of London B: Biological Sciences, 275, 2651-2656.

Carré, J. M., McCormick, C. M., \& Mondloch, C. J. (2009). Facial structure is a reliable cue of aggressive behavior. Psychological Science, 20, 1194-1198.

Carré, J. M., Murphy, K. R., \& Hariri, A. R. (2013). What lies beneath the face of aggression?. Social Cognitive and Affective Neuroscience, 8, 224-229.

Castano, E., \& Giner-Sorolla, R. (2006). Not quite human: Infrahumanization in response to collective responsibility for intergroup killing. Journal of Personality and Social Psychology, 90, 804-818.

Cooley, E., Payne, B. K., Cipolli, W., Cameron, D, Berger, A., \& Gray, K. (2017). The paradox of group mind: "People in a group" have more mind than "a group of people." Journal of Experimental Psychology: General, 146, 691-699.

Cortes, B. P., Demoulin, S., Rodriguez, R. T., Rodriguez, A. P., \& Leyens, J. P. (2005). Infrahumanization or familiarity? Attribution of uniquely human emotions to the self, the ingroup, and the outgroup. Personality and Social Psychology Bulletin, 31, 243-253.

Cuddy, A. J., Rock, M. S., \& Norton, M. I. (2007). Aid in the aftermath of Hurricane Katrina: Inferences of secondary emotions and intergroup helping. Group Processes \& Intergroup Relations, 10, 107-118.

Deaner, R. O., Goetz, S. M., Shattuck, K., \& Schnotala, T. (2012). Body weight, not facial width-to-height ratio, predicts aggression in pro hockey players. Journal of Research in Personality, 46, 235-238. 
Deska, J. C., Almaraz, S. M., \& Hugenberg, K. (2017). Of mannequins and men: Judgments of animacy in faces are bounded by perceptual and processing similarities to human faces. Social Psychological and Personality Science, 8, 183-190.

Deska, J. C., Lloyd, E. P., \& Hugenberg, K. (in press). The face of fear and anger: Facial widthto-height ratio biases recognition of angry and fearful expressions. Emotion.

Dion, K., Berscheid, E., \& Walster, E. (1972). What is beautiful is good. Journal of Personality and Social Psychology, 24, 285.

Dovidio, J. F., \& Gaertner, S. L. (1999). Reducing prejudice combating intergroup biases. Current Directions in Psychological Science, 8, 101-105.

Epley, N., Akalis, S., Waytz, A., \& Cacioppo, J. T. (2008). Creating social connection through inferential reproduction: Loneliness and perceived agency in gadgets, gods, and greyhounds. Psychological Science, 19, 114-120.

Epley, N., Schroeder, K., \& Waytz, A. (2013). Motivated mind perception: Treating pets as people and people as animals. In S. J. Gervais (ed.), Nebraska Symposium on Motivation (Vol. 60, pp 127-152). New York, NY: Springer.

Epley, N., Waytz, A., Akalis, S., \& Cacioppo, J. T. (2008). When we need a human: Motivational determinants of anthropomorphism. Social Cognition, 26, 143-155.

Epley, N., Waytz, A., \& Cacioppo, J. T. (2007). On seeing human: A three-factor theory of anthropomorphism. Psychological Review, 114, 864-886.

Fiedler, K., Schott, M., \& Meiser, T. (2011). What mediation analyses can (not) do. Journal of Experimental Social Psychology, 47, 1231-1236. 
Fincher, K. M., \& Tetlock, P. E. (2016). Perceptual dehumanization of faces is activated by norm violations and facilitates norm enforcement. Journal of Experimental Psychology: General, 145, 131-146.

Fredrickson, B. L., \& Roberts, T. A. (1997). Objectification theory. Psychology of Women Quarterly, 21, 173-206.

Freeman, J. B., \& Ambady, N. (2011). A dynamic interactive theory of person construal. Psychological Review, 118, 247-279.

Geniole, S. N., Denson, T. F., Dixson, B. J., Carré, J. M., \& McCormick, C. M. (2015). Evidence from meta-analyses of the facial width-to-height ratio as an evolved cue of threat. PLoS ONE, 10, e0132726.

Geniole, S. N., Keyes, A. E., Carré, J. M., \& McCormick, C. M. (2014). Fearless dominance mediates the relationship between the facial width-to-height ratio and willingness to cheat. Personality and Individual Differences, 57, 59-64.

Goff, P. A., Eberhardt, J. L., Williams, M. J., \& Jackson, M. C. (2008). Not yet human: Implicit knowledge, historical dehumanization, and contemporary consequences. Journal of Personality and Social Psychology, 94, 292-306.

Goff, P. A., Jackson, M. C., Di Leone, B. A. L., Culotta, C. M., \& DiTomasso, N. A. (2014). The essence of innocence: Consequences of dehumanizing Black children. Journal of Personality and Social Psychology, 106, 526-545.

Goh, J. X., Hall, J. A., \& Rosenthal, R. (2016). Mini meta-analysis of your own studies: Some arguments on why and a primer on how. Social and Personality Psychology Compass, $10,535-549$. 
Golby, A. J., Gabrieli, J. D., Chiao, J. Y., \& Eberhardt, J. L. (2001). Differential responses in the fusiform region to same-race and other-race faces. Nature Neuroscience, 4, 845-850.

Gray, H. M., Gray, K., \& Wegner, D. M. (2007). Dimensions of mind perception. Science, 315, 619-619.

Gray, K., Knobe, J., Sheskin, M., Bloom, P., \& Barrett, L. F. (2011). More than a body: Mind perception and the nature of objectification. Journal of Personality and Social Psychology, 101, 1207-1220.

Gray, K., \& Wegner, D. M. (2010). Blaming God for our pain: Human suffering and the divine mind. Personality and Social Psychology Review, 14, 7-16.

Gray, K., Young, L., \& Waytz, A. (2012). Mind perception is the essence of morality. Psychological Inquiry, 23, 101-124.

Gruenfeld, D. H., Inesi, M. E., Magee, J. C., \& Galinsky, A. D. (2008). Power and the objectification of social targets. Journal of Personality and Social Psychology, 95, 111127.

Haque, O. S., \& Waytz, A. (2012). Dehumanization in medicine: Causes, solutions, and functions. Perspectives on Psychological Science, 7, 176-186.

Haselhuhn, M. P., Ormiston, M. E., \& Wong, E. M. (2015). Men's facial width-to-height ratio predicts aggression: A meta-analysis. PLoS ONE, 10, e0122637.

Haslam, N. (2006). Dehumanization: An integrative review. Personality and Social Psychology Review, 10, 252-264.

Haslam, N. (2014). What is dehumanization? In P. G. Bain, J. Vaes, \& J.-P. Leyens (Eds.), Humanness and dehumanization (pp. 34-48). New York, NY: Taylor \& Francis. 
Haslam, N., \& Bain, P. (2007). Humanizing the self: Moderators of the attribution of lesser humanness to others. Personality and Social Psychology Bulletin, 33, 57-68.

Haslam, N., Bain, P., Douge, L., Lee, M., \& Bastian, B. (2005). More human than you: Attributing humanness to self and others. Journal of Personality and Social Psychology, 89, 937-950.

Haslam, N. \& Loughnan, S. (2012). Dehumanization and prejudice. In J. Dixon \& M. Levine (Eds.), Beyond prejudice: Extending the social psychology of intergroup conflict, inequality and social change (pp. 89-104). Cambridge University Press

Haslam, N., \& Loughnan, S. (2014). Dehumanization and infrahumanization. Annual Review of Psychology, 65, 399-423.

Hehman, E., Flake, J. K., \& Freeman, J. B. (2015). Static and dynamic facial cues differentially affect the consistency of social evaluations. Personality and Social Psychology Bulletin, $41,1123-1134$.

Hehman, E., Leitner, J. B., Deegan, M. P., \& Gaertner, S. L. (2013). Facial structure is indicative of explicit support for prejudicial beliefs. Psychological Science, 24, 289-296.

Hehman, E., Leitner, J. B., Deegan, M. P., \& Gaertner, S. L. (2015). Picking teams: When dominant facial structure is preferred. Journal of Experimental Social Psychology, 59, $51-59$.

Hodges-Simeon, C. R., Sobraske, K. N. H., Samore, T., Gurven, M., \& Gaulin, S. J. (2016). Facial width-to-height ratio (fWHR) is not associated with adolescent testosterone levels. PLoS ONE, 11, e0153083. 
Hugenberg, K., \& Wilson, J. P. (2013). Faces are central to social cognition. In D. Carlston (Ed.), Handbook of Social Cognition (pp. 167-193). New York, NY: Oxford University Press.

Hugenberg, K., Young, S., Rydell, R. J., Almaraz, S., Stanko, K. A., See, P. E., \& Wilson, J. P. (2016). The face of humanity: Configural face processing influences ascriptions of humanness. Social Psychological and Personality Science, 7, 167-175.

Iverson, R. D., \& Maguire, C. (2000). The relationship between job and life satisfaction: Evidence from a remote mining community. Human Relations, 53, 807-839.

Judd, C. M., Westfall, J., \& Kenny, D. A. (2012). Treating stimuli as a random factor in social psychology: A new and comprehensive solution to a pervasive but largely ignored problem. Journal of Personality and Social Psychology, 103, 54-69.

Judd, C. M., Westfall, J., \& Kenny, D. A. (2017). Experiments with more than one random factor: Designs, analytic models, and statistical power. Annual Review of Psychology, 68, $601-625$.

Kelman, H. C. (1976). Violence without restraint: Reflections on the dehumanization of victims and victimizers. In G. M. Kren \& L. H. Rappoport (Eds.), Varieties of Psychohistory (pp. 282-314). New York: Springer.

Khalid, S., Deska, J. C., \& Hugenberg, K. (2016). The eyes are the windows to the mind: Direct eye gaze triggers the ascription of others' minds. Personality and Social Psychology Bulletin, 42, 1666-1677.

Kteily, N., \& Bruneau, E. (in press). Darker demons of our nature: The need to (re-)focus attention on blatant forms of dehumanization. Current Direction in Psychological Science. 
Kteily, N., Bruneau, E., Waytz, A., \& Cotterill, S. (2015). The ascent of man: Theoretical and empirical evidence for blatant dehumanization. Journal of Personality and Social Psychology, 109, 901-931.

Kteily, N., Hodson, G., \& Bruneau, E. (2016). They see us as less than human: Metadehumanization predicts intergroup conflict via reciprocal dehumanization. Journal of Personality and Social Psychology, 110, 343-370.

Kramer, R. S., Jones, A. L., \& Ward, R. (2012). A lack of sexual dimorphism in width-to-height ratio in white European faces using 2D photographs, 3D scans, and anthropometry. PLoS ONE, 7, e42705.

Lefevre, C. E., Etchells, P. J., Howell, E. C., Clark, A. P., \& Penton-Voak, I. S. (2014). Facial width-to-height ratio predicts self-reported dominance and aggression in males and females, but a measure of masculinity does not. Biology Letters, 10, 20140729.

Lefevre, C. E., Lewis, G. J., Perrett, D. I., \& Penke, L. (2013). Telling facial metrics: Facial width is associated with testosterone levels in men. Evolution and Human Behavior, 34, 273-279.

Leyens, J. P., Demoulin, S., Vaes, J., Gaunt, R., \& Paladino, M. P. (2007). Infra-humanization: The wall of group differences. Social Issues and Policy Review, 1, 139-172.

Leyens, J. P., Paladino, P. M., Rodriguez-Torres, R., Vaes, J., Demoulin, S., Rodriguez-Perez, A., \& Gaunt, R. (2000). The emotional side of prejudice: The attribution of secondary emotions to ingroups and outgroups. Personality and Social Psychology Review, 4, 186197.

Looser, C. E., \& Wheatley, T. (2010). The tipping point of animacy: How, when, and where we perceive life in a face. Psychological Science, 21, 1854-1862. 
Loughnan, S., \& Haslam, N. (2007). Animals and androids: Implicit associations between social categories and nonhumans. Psychological Science, 18, 116-121.

Lyubomirsky, S., \& Lepper, H. S. (1999). A measure of subjective happiness: Preliminary reliability and construct validation. Social Indicators Research, 46, 137-155.

Ma, D. S., Correll, J., \& Wittenbrink, B. (2015). The Chicago face database: A free stimulus set of faces and norming data. Behavior Research Methods, 47, 1122-1135.

Macrae, C. N., \& Shepherd, J. W. (1989). Do criminal stereotypes mediate juridic judgements?. British Journal of Social Psychology, 28, 189-191.

MacKinnon, D. P., Lockwood, C. M., \& Williams, J. (2004). Confidence limits for the indirect effect: Distribution of the product and resampling methods. Multivariate Behavioral Research, 39, 99-128.

Mileva, V. R., Cowan, M. L., Cobey, K. D., Knowles, K. K., \& Little, A. C. (2014). In the face of dominance: Self-perceived and other-perceived dominance are positively associated with facial-width-to-height ratio in men. Personality and Individual Differences, 69, 115118.

Montepare, J. M., \& Zebrowitz, L. A. (1998). Person perception comes of age: The salience and significance of age in social judgments. Advances in Experimental Social Psychology, 30, 93-161.

Neth, D., \& Martinez, A. M. (2010). A computational shape-based model of anger and sadness justifies a configural representation of faces. Vision Research, 50, 1693-1711.

Nussbaum, M. C. (1999). Sex and social justice. New York: Oxford University Press.

Oosterhof, N. N., \& Todorov, A. (2008). The functional basis of face evaluation. Proceedings of the National Academy of Sciences, 105, 11087-11092. 
Oosterhof, N. N., \& Todorov, A. (2009). Shared perceptual basis of emotional expressions and trustworthiness impressions from faces. Emotion, 9, 128-133.

Opotow, S. (1990). Moral exclusion and injustice: An introduction. Journal of Social Issues, 46, $1-20$.

Özener, B. (2012). Facial width-to-height ratio in a Turkish population is not sexually dimorphic and is unrelated to aggressive behavior. Evolution and Human Behavior, 33, 169-173.

Pavot, W., \& Diener, E. (2008). The satisfaction with life scale and the emerging construct of life satisfaction. The Journal of Positive Psychology, 3, 137-152.

Pereira, C., Vala, J., \& Leyens, J. P. (2009). From infra-humanization to discrimination: The mediation of symbolic threat needs egalitarian norms. Journal of Experimental Social Psychology, 45, 336-344.

Powers, K. E., Worsham, A. L., Freeman, J. B., Wheatley, T., \& Heatherton, T. F. (2014). Social connection modulates perceptions of animacy. Psychological Science, 25, 1943-1948.

Schönbrodt, F. D. (2015). p-checker: One-for-all p-value analyzer. Retrieved from http://shinyapps.org/apps/p-checker/.

Schroeder, J. \& Epley, N. (2015). The sound of intellect: Speech reveals a thoughtful mind, increasing a job candidate's appeal. Psychological Science, 26, 877-891.

Short, L. A., Mondloch, C. J., McCormick, C. M., Carré, J. M., Ma, R., Fu, G., \& Lee, K. (2012). Detection of propensity for aggression based on facial structure irrespective of face race. Evolution and Human Behavior, 33, 121-129.

Stirrat, M., \& Perrett, D. I. (2010). Valid facial cues to cooperation and trust: Male facial width and trustworthiness. Psychological Science, 21, 349-354. 
Thoemmes, F. (2015). Reversing arrows in mediation models does not distinguish plausible models. Basic and Applied Social Psychology, 37, 226-234.

Todorov, A., Said, C. P., Engell, A. D., \& Oosterhof, N. N. (2008). Understanding evaluation of faces on social dimensions. Trends in Cognitive Sciences, 12, 455-460.

Trawalter, S., Hoffman, K. M., \& Waytz, A. (2012). Racial bias in perceptions of others' pain. PLoS ONE, 7, e48546.

Vaes, J., Bain, P. G., \& Leyens, J. P. (2014) Understanding humanness and dehumanization: Emerging themes and directions. In Bain, P., Vaes, J., \& Leyens, J. P. (Eds.) Humanness and Dehumanization (pp. 323-335. Taylor \& Francis, New York).

Vaes, J., Paladino, M. P., Castelli, L., Leyens, J. P., \& Giovanazzi, A. (2003). On the behavioral consequences of infrahumanization: The implicit role of uniquely human emotions in intergroup relations. Journal of Personality and Social Psychology, 85, 1016.

Veenhoven, R. (1996). Developments in satisfaction-research. Social Indicators Research, 37, 146.

Viki, G. T., Osgood, D., \& Phillips, S. (2013). Dehumanization and self-reported proclivity to torture prisoners of war. Journal of Experimental Social Psychology, 49, 325-328.

Waytz, A., Gray, K., Epley, N., \& Wegner, D. M. (2010). Causes and consequences of mind perception. Trends in Cognitive Sciences, 14, 383-388.

Waytz, A., Morewedge, C. K., Epley, N., Monteleone, G., Gao, J. H., \& Cacioppo, J. T. (2010). Making sense by making sentient: Effectance motivation increases anthropomorphism. Journal of Personality and Social Psychology, 99, 410-435.

Weston, E. M., Friday, A. E., \& Liò, P. (2007). Biometric evidence that sexual selection has shaped the hominin face. PLoS ONE, 2, e710. 
Wilson, J. P., \& Rule, N. O. (2015). Facial trustworthiness predicts extreme criminal-sentencing outcomes. Psychological Science, 26, 1325-1331.

Wittgenstein, L., \& Anscombe, G. E. M. (1958). Philosophical Investigations. Oxford: Blackwell.

Young, S. G., Slepian, M. L., Wilson, J. P., \& Hugenberg, K. (2014). Averted eye-gaze disrupts configural face encoding. Journal of Experimental Psychology, 53, 94-99.

Zebrowitz, L. A. (1997). Reading faces: Window to the soul? Boulder, CO: Westview Press.

Zebrowitz, L. A., \& Montepare, J. M. (1992). Impressions of babyfaced individuals across the life span. Developmental Psychology, 28, 1143-1152.

Zebrowitz, L. A., \& Montepare, J. M. (2008). Social psychological face perception: Why appearance matters. Social and Personality Psychology Compass, 2, 1497-1517. 

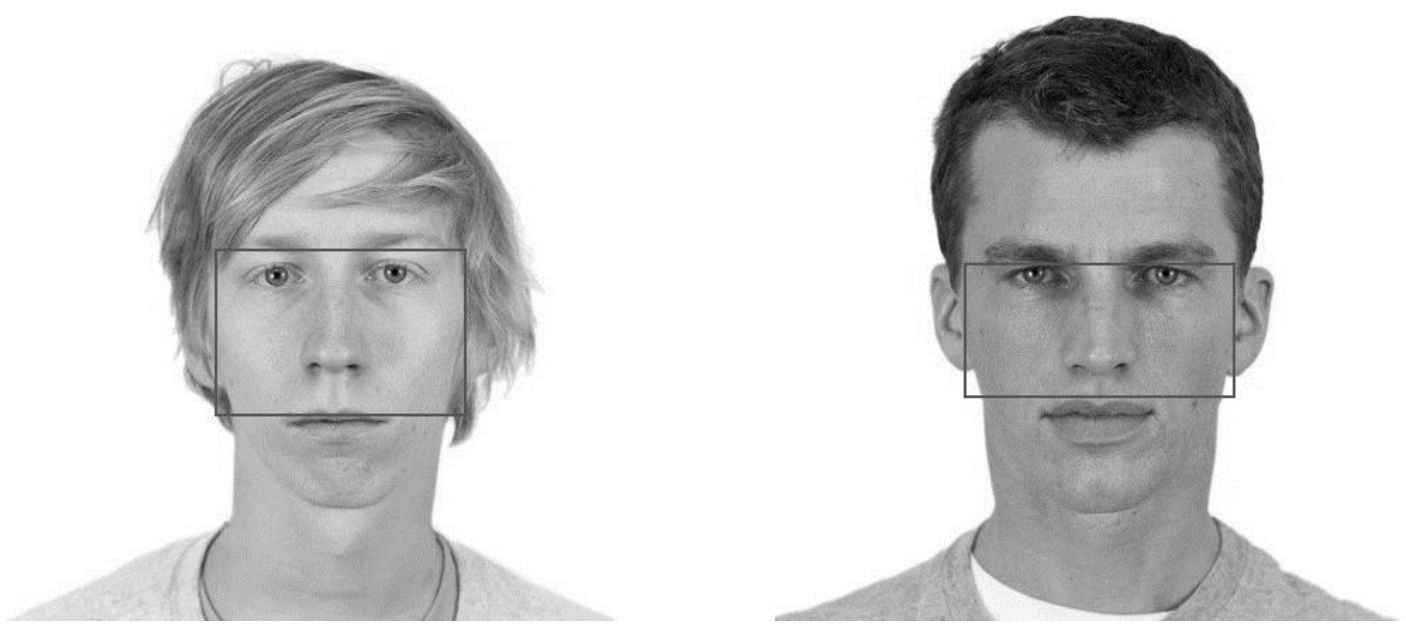

Figure 1. Facial width-to-height ratio is commonly measured as the ratio between bizygomatic width (i.e., distance between left to right zygion) to upper face height (i.e., distance between mid-brow and upper lip. 


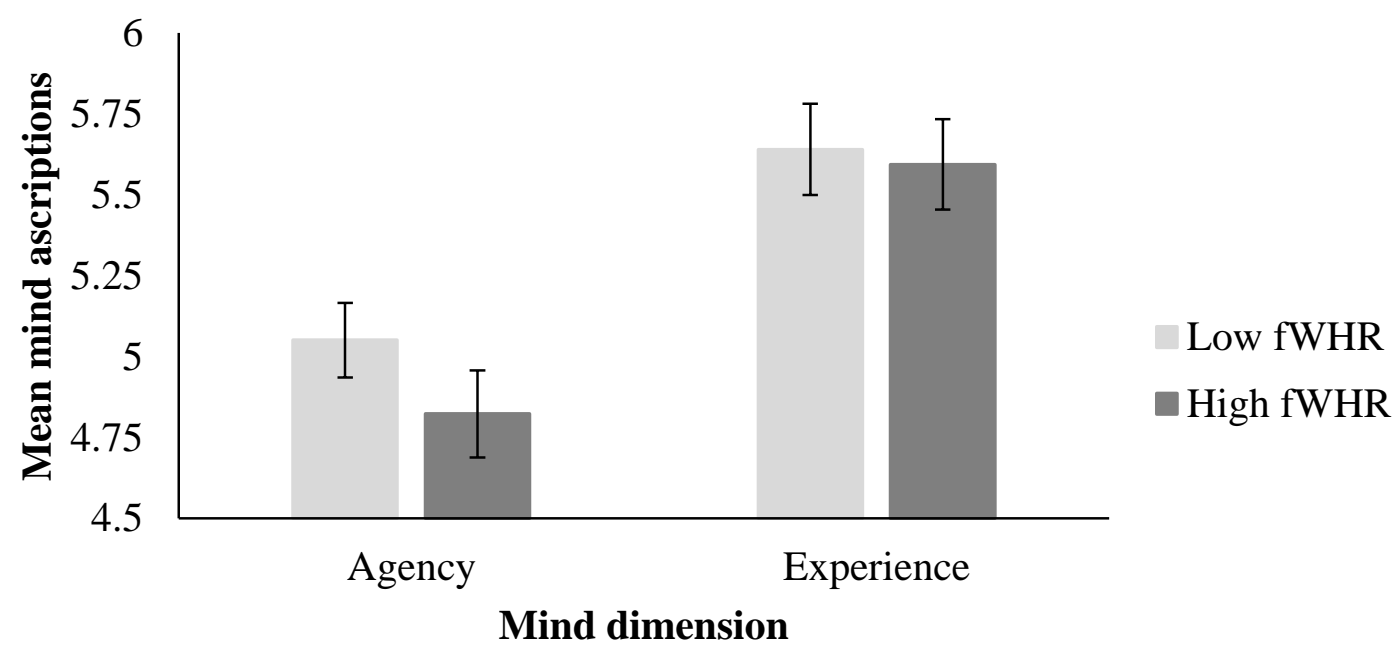

Figure 2: Mean mind ascription as a function of dimension of mind and fWHR in Study 2. High fWHR targets were denied agency, but not experience, relative to low fWHR targets. Error bars represent standard error of the mean. 


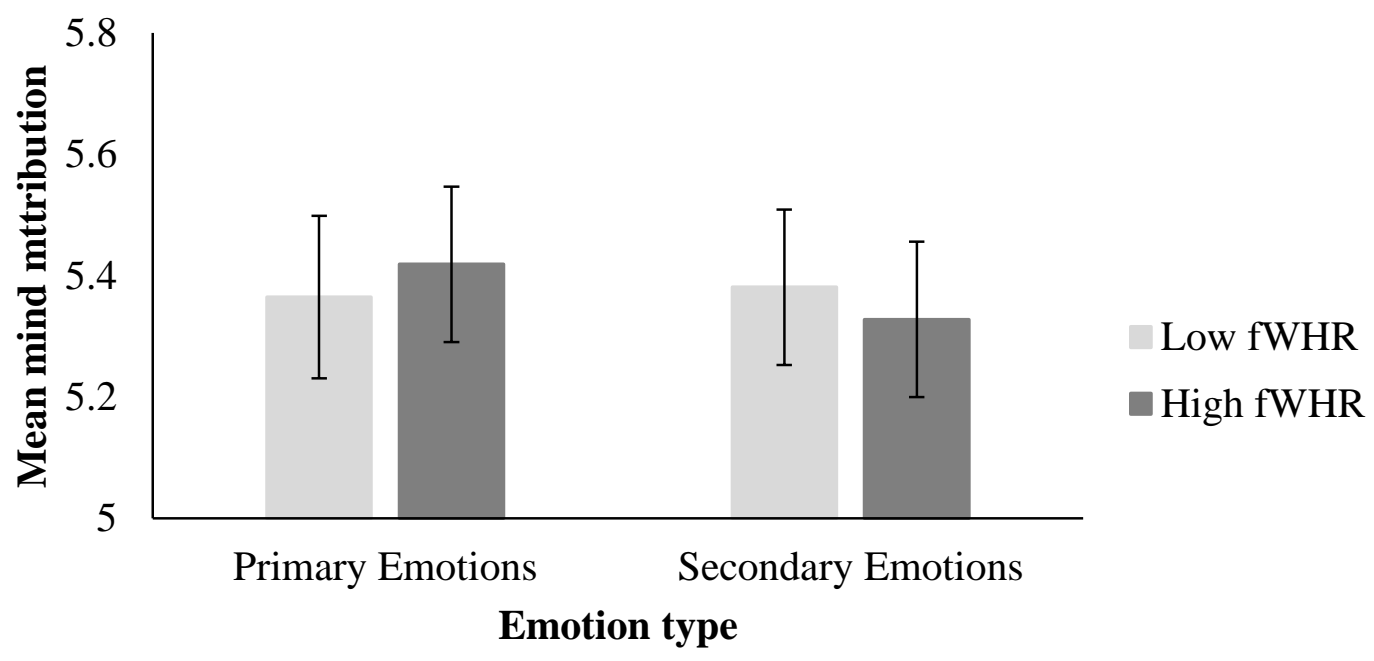

Figure 3: Mean ascriptions of emotional capacity as a function of emotion type and fWHR in Study 3a. High fWHR targets were perceived to be less capable of experiencing secondary, but not primary, emotions than low fWHR targets. Error bars represent standard error of the mean. 


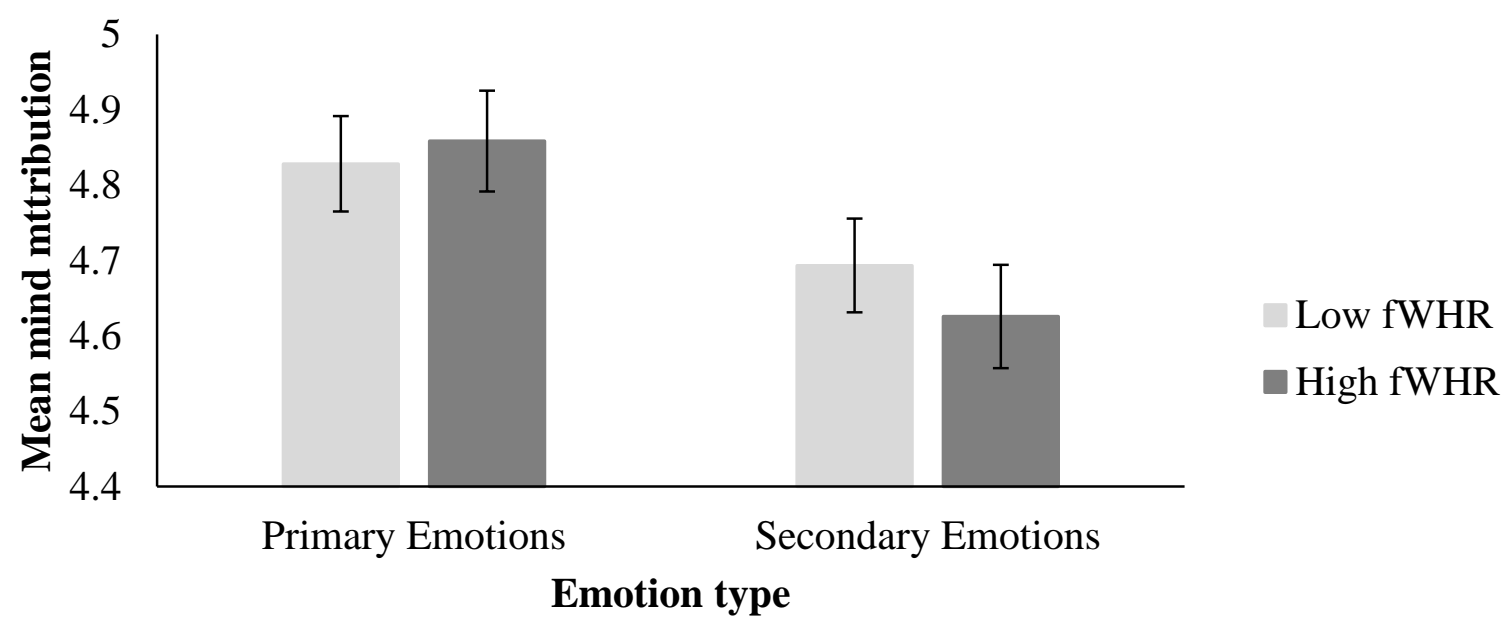

Figure 4: Mean ascriptions of emotional capacity as a function of emotion type and fWHR in Study 3b. High fWHR targets were perceived to be less capable of experiencing secondary, but not primary, emotions than low fWHR targets. Error bars represent standard error of the mean. 


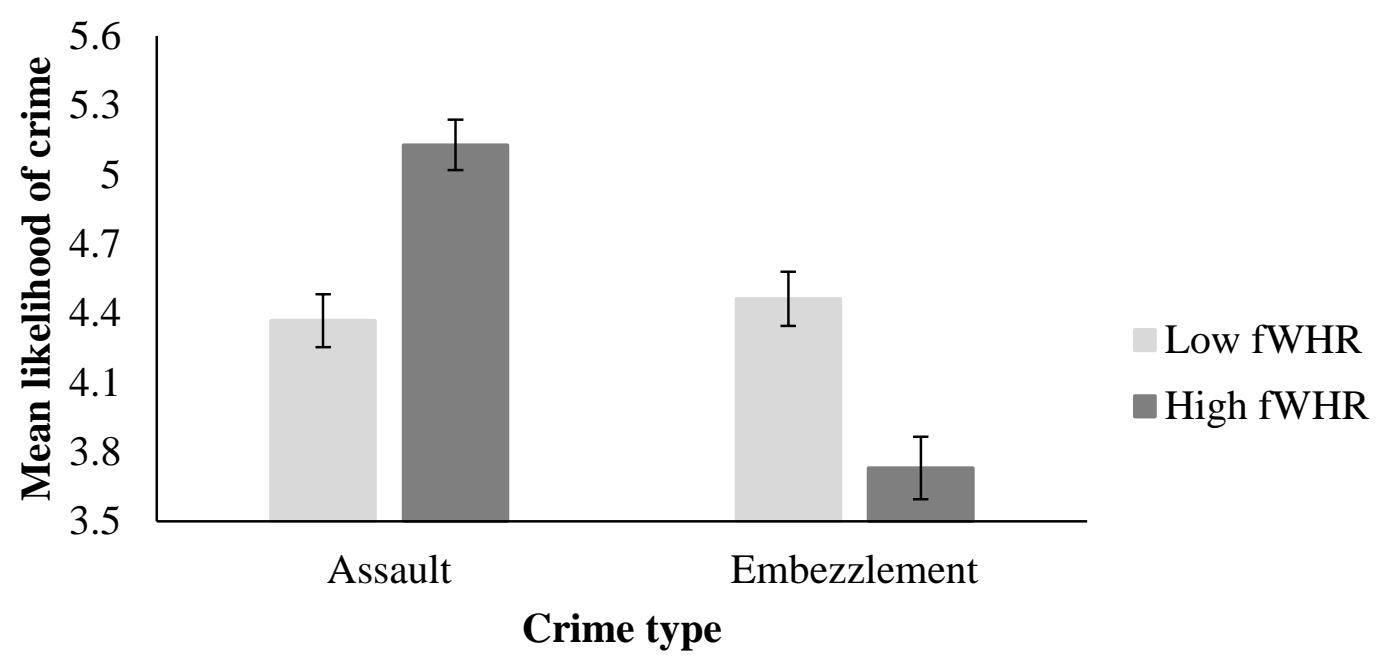

Figure 5: Perceptions of the likelihood of committing a crime as a function of crime type and fWHR in Study 6. High fWHR targets were perceived as guiltier of assault than low fWHR targets, who were in turn seen as guiltier of embezzlement. Error bars represent standard error of the mean. 


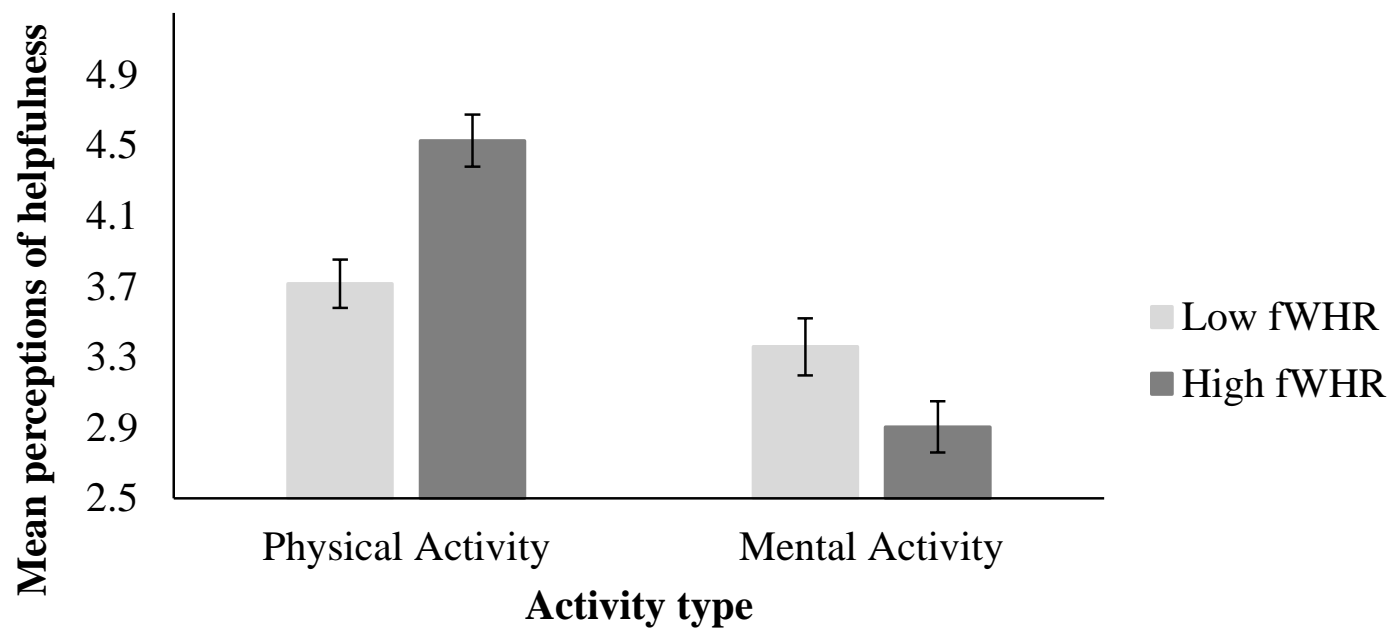

Figure 6: Perceptions of helpfulness as a function of activity type and fWHR in Study 7. Although both high and low fWHR targets were perceived as more helpful for physical activities than mental activities, this was particularly true for high fWHR compared to low fWHR targets. Error bars represent standard error of the mean. 


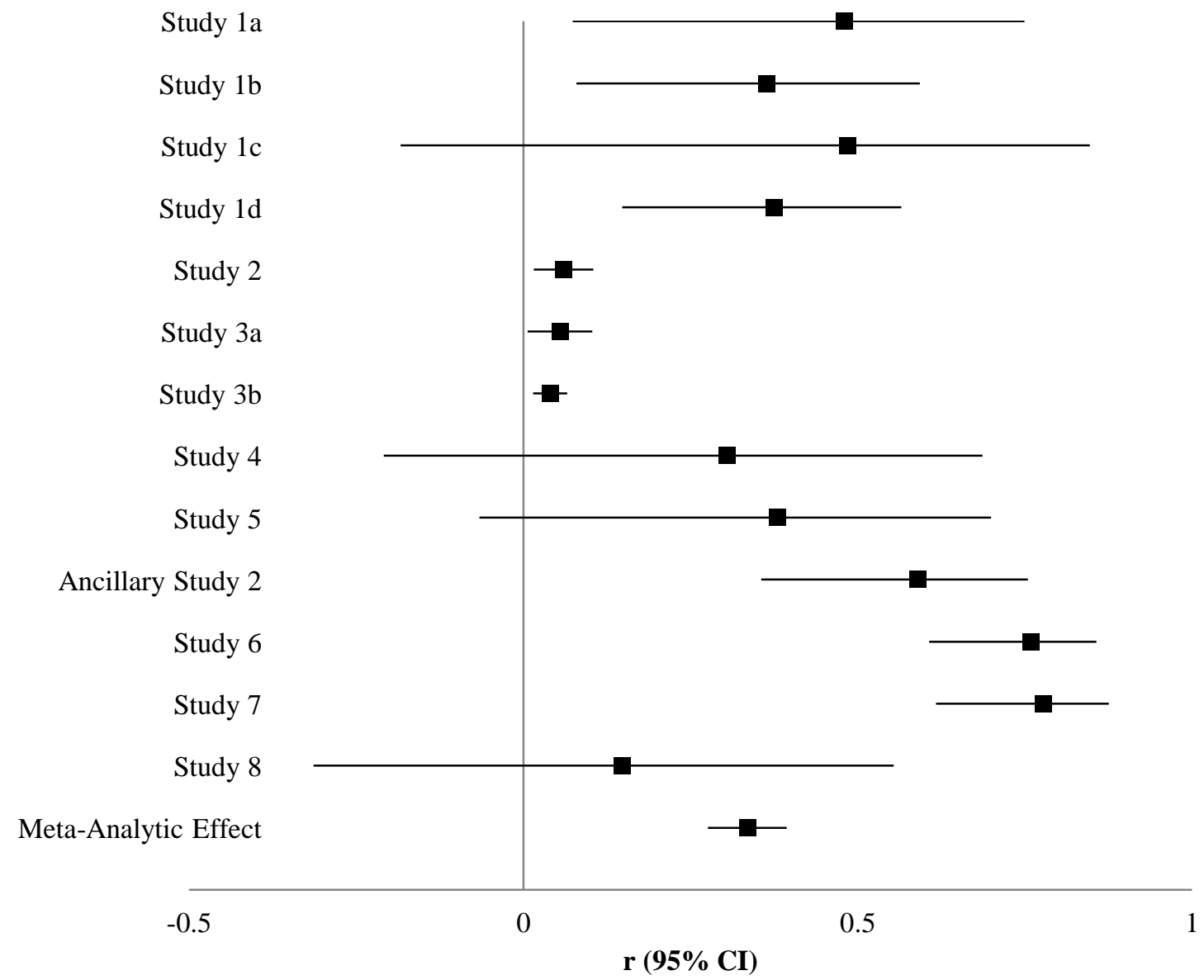

Figure 7: Forest plot depicting the effect size $r$ from each study as well as the overall metaanalytic effect. Error bars represent 95\% confidence intervals around the effect size $r$. 